\title{
Surface structure of $\mathrm{SrTiO}_{3}(001)$
}

\author{
R. Herger, P. R. Willmott,* O. Bunk, C. M. Schlepütz, and B. D. Patterson \\ Swiss Light Source, Paul Scherrer Institut, CH-5232 Villigen, Switzerland \\ B. Delley \\ Condensed Matter Theory Group, Paul Scherrer Institut, CH-5232 Villigen, Switzerland
}

\begin{abstract}
V. L. Shneerson, P. F. Lyman, and D. K. Saldin
Department of Physics and the Laboratory for Surface Studies, University of Wisconsin-Milwaukee, Milwaukee, Wisconsin 53201, USA
\end{abstract}

(Received 6 June 2007; published 21 November 2007)

\begin{abstract}
We report on the structural determination of the surface of $\mathrm{TiO}_{2}$-terminated $\mathrm{SrTiO}_{3}(001)$ using surface x-ray diffraction. The detailed analysis of two surface diffraction data sets are presented, one (cold) taken at room temperature in vacuum, and the other (hot) under typical conditions used for thin film growth. 49 different combinations of possible surface terminations are described for the cold structure, from which the final structure was chosen, consisting of a weighted mixture of a $(1 \times 1)$ relaxation and $(2 \times 1)$ and $(2 \times 2)$ reconstructions, simultaneously present at the surface. The structures are best modeled by a $\mathrm{TiO}_{2}$-rich surface similar to that proposed by Erdman et al. [Nature (London) 419, 55 (2002)]. The reconstructions are energetically favorable according to density functional theory. They disappear within several minutes upon heating to the hot conditions, forming a termination very similar to the cold $(1 \times 1)$, but more puckered and higher in energy. Six additional models, suggested by direct methods and the literature, to describe the hot surface are also discussed. Direct methods confirm the $\mathrm{TiO}_{2}$-rich termination and the atomic positions of the hot surface. The atomic coordinates for the two $\mathrm{TiO}_{2}$-rich surfaces exhibit significant displacements down to three unit cells, which may have important implications on possible surface ferroelectric phenomena in $\mathrm{SrTiO}_{3}$. Surface energy considerations suggest a temperature-induced order-disorder transition, produced by a mixing of the $(2 \times 1)$ and $(2 \times 2)$ reconstructions, to form the hot pseudo $(1 \times 1)$ structure. Electrostatic stability arguments provide circumstantial support for the experimentally determined $\mathrm{TiO}_{2}$-rich surfaces.
\end{abstract}

DOI: 10.1103/PhysRevB.76.195435 PACS number(s): 61.10.Nz, 68.35.Bs, 68.47.Gh, 71.15.Mb

\section{INTRODUCTION}

Perovskites have been subject of intense research in recent years due to their intriguing physical properties, such as high-temperature superconductivity, colossal magnetoresistance, ferroelectricity, and heterostructures containing twodimensional electron gases. ${ }^{1-4}$ The potential technological applications of perovskites in mass storage devices, as read heads or ferroelectric random access memories, to mention just two examples, underline the importance of understanding the atomic structures of such complex metal oxides. Because in most applications, these materials are used in the form of thin films, it is especially important that their surface and interfacial structures are understood. Subtle structural differences in these regions may lead to fundamentally different physical properties, due to the strong correlation of the valence electrons. On the one hand, surface effects such as reconstructions can set a lower limit to downsizing of devices that exploit bulk effects, while on the other, unexpected new phenomena, for example, ferroelectricity, may occur at the surface. ${ }^{2,5}$

Strontium titanate $\left(\mathrm{SrTiO}_{3}, \mathrm{STO}\right)$ is the paradigmatic substrate material for thin film growth of perovskites. While the surface of STO has been the subject of intense investigations, there remains a notable lack of a detailed description with sub- $\AA$ resolution after typical ex situ chemical and thermal preparation and, subsequently, under thin film growth conditions. As a result, no concise picture of the surface structure exists, and thus, the STO surface remains a controversial subject. This is partly due on the one hand to the sensitivity of the surface to the preparation and processing conditions, and on the other, to the limited spatial resolution associated with most surface characterization techniques. Consequently, at least nine different surface terminations have been reported for $\mathrm{STO}(001)$, depending on the various preparation and ambient conditions, including $(1 \times 1),(2 \times 1),(2 \times 2)$, $(6 \times 2), c(4 \times 2), c(6 \times 2), c(4 \times 4),(\sqrt{5} \times \sqrt{5}) R 26.6^{\circ}$, and $(\sqrt{13} \times \sqrt{13}) R 33.7^{\circ}$ reconstructions and relaxations. ${ }^{6-19}$ Of these observations, two report the simultaneous presence of more than one surface termination at the same time., ${ }^{9,19}$

Of particular relevance among the above terminations to this Report is a Ti-rich $(2 \times 1)$ reconstruction proposed by Erdman et al..${ }^{14}$ where the surface terminates not with one, but two $\mathrm{TiO}_{2}$ atomic layers (ALs), determined by transmission electron microscopy. The topmost Ti atoms form a characteristic "zigzag" motif, while the Ti sites in the next AL, i.e., one layer deeper down, occupy bulk positions. $\mathrm{This}^{\mathrm{TiO}_{2}}$ double layer (DL) structure, and adaptations of it, have been widely discussed. ${ }^{12,15,17,19,20}$

We recently reported on coexisting DL terminations on STO, consisting of a $(2 \times 1)$ reconstruction similar to that described by Erdman et al., ${ }^{14}$ plus a novel $(2 \times 2)$ reconstruction, as well as a $\mathrm{TiO}_{2}(1 \times 1)$ DL relaxation on the surface of STO. ${ }^{21}$ In this work, we present a detailed account of the many models we considered for the large surface X-ray diffraction (SXRD) data set of the STO(001) surface that we acquired, and how we finally determined the DL model de- 


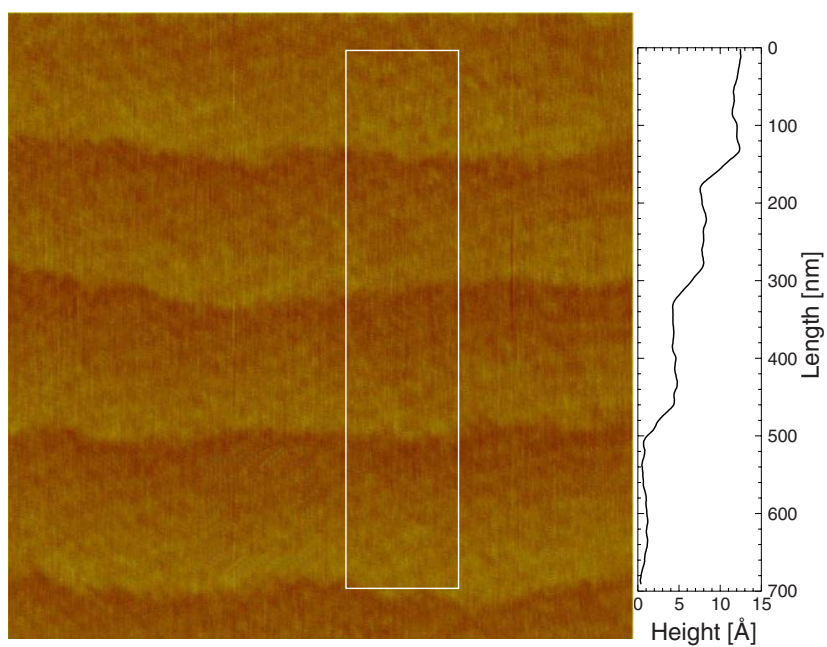

FIG. 1. (Color online) AFM image of STO after chemical etching and thermal annealing. The step height of $4 \AA$ corresponds well to a unit cell step, while no $2 \AA$ steps, indicative of mixed $\mathrm{SrO} / \mathrm{TiO}_{2}$ termination, were observed. The line profile (right) was generated by horizontal integration over the region marked by the white box.

scribed in Ref. 21. In arriving this model, we were guided by complementary experimental data, possible models suggested by phase-retrieval methods, and our own theoretical calculations, all of which are also described here. Structures were refined for two data sets, the first taken at room temperature and in ultrahigh vacuum (henceforth referred to as "cold"), and the second recorded at elevated temperature and in an oxygen atmosphere, typical for the growth of perovskite thin films (henceforth referred to as "hot").

\section{METHODS}

\section{A. Experimental}

\section{Substrate preparation}

STO(001) substrates (space group $P m \overline{3} m, a_{0}=3.9045 \AA$ ) with low vicinality $\left(<0.1^{\circ}\right)$ and an impurity content of $<5 \mathrm{ppm} \mathrm{Ca}$ (Ref. 22) were purchased from CrysTec $\mathrm{GmbH}$, Germany. Substrates of $10 \times 8 \times 1 \mathrm{~mm}^{3}$ were prepared according to an established chemical and thermal treatment, ${ }^{23,24}$ in order to ensure $100 \%$ termination on the $\mathrm{TiO}_{2}$ $\mathrm{AL}$ and smooth terrace edge profiles.

\section{Characterization}

Atomic force microscopy (AFM, Fig. 1) performed on prepared STO revealed a uniform terrace step height of about $4 \AA$ (i.e., one unit cell). Comparison with AFM images of unannealed surfaces (not shown) confirmed that annealing smooths the terrace-edge contours. The terraces are $200 \mathrm{~nm}$ wide, consistent with the nominal vicinality.

$\mathrm{X}$-ray photoelectron spectroscopy (XPS) using an $\mathrm{Al} K \alpha$ source at $1487 \mathrm{eV}$ was used to confirm $\mathrm{TiO}_{2}$ termination. The angle-dependent electron yield of the oxygen $1 s$ signal showed two peaks that could be assigned to bulk and surface

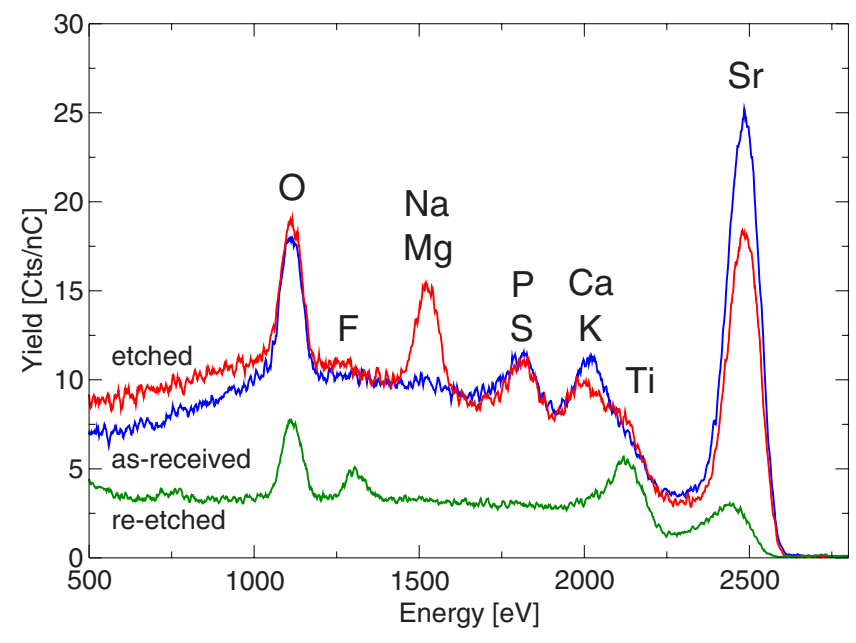

FIG. 2. (Color online) LEIS measurements of as-received (blue) and etched (red) (Refs. 23 and 24) and re-etchted STO (green) (Ref. 27). In addition to $\mathrm{Sr}$, $\mathrm{Ti}$, and $\mathrm{O}$, also $\mathrm{Ca} / \mathrm{K}, \mathrm{P} / \mathrm{S}, \mathrm{Na} / \mathrm{Mg}$, and $\mathrm{F}$ are present in the outermost layer. The spectra show decreased intensities for the $\mathrm{Sr}$ and $\mathrm{Ca}$ species, but an enhanced $\mathrm{Ti}$ signal for prepared STO. The main contaminant $\mathrm{Mg}$ could be explained by adsorbates arising from the $\mathrm{MgO}$ crucible during the anneal process.

contributions. Due to the insulating properties of STO, only relative shifts in energy, i.e., the chemical shifts, could be established. The chemical shifts between the bulk and surface contributions were 2.6 and $2.0 \mathrm{eV}$ for as-received and prepared STO, respectively. This is consistent with the chemical shifts found for $\mathrm{SrO}(2.7 \mathrm{eV})$ and $\mathrm{TiO}_{2}(1.9 \mathrm{eV}){ }^{25}$ This suggests a $\mathrm{TiO}_{2}$ termination for etched $\mathrm{STO}$, whereas for unprepared samples both $\mathrm{TiO}_{2}$ and $\mathrm{SrO}$ terminations are present.

Low-energy ion scattering (LEIS) was performed by Calipso B.V. at the Technical University of Eindhoven, the Netherlands. ${ }^{26} \mathrm{The}^{4} \mathrm{He}^{+}$ions had an energy of $3 \mathrm{keV}$. In Fig. 2 , we present the LEIS spectra of as-received STO and STO prepared according to Refs. 23, 24, and 27. The red and the blue spectra were taken after exposing the samples to atomic oxygen of thermal energy for $20 \mathrm{~min}$, in order to remove environmental pollution. The green spectrum was taken after an additional etch and rinse step of the etched sample (i.e., of the one that gave rise to the red LEIS spectrum). One or two chemical elements are assigned to each of the peaks, depending on whether the measurement could adequately separate the masses. Section III D will further discuss the LEIS results shown in Fig. 2.

\section{Surface $x$-ray diffraction}

SXRD experiments were carried out at the surface diffraction station of the Materials Science Beamline at the Swiss Light Source, Paul Scherrer Institut. ${ }^{28}$ The substrates were introduced via a vacuum loadlock into an ultrahigh vacuum (UHV) chamber equipped with a large beryllium window ${ }^{29}$ mounted on a $(2+3)$ circle surface diffractometer. Owing to the availability of a fast single photon-counting twodimensional x-ray pixel detector, it was feasible to reliably record a data set of independent structure factors which was 
sufficiently large to refine a highly complex structural model within a limited beamtime. From the pixel images, the integrated intensities were extracted and the standard geometrical correction factors applied. ${ }^{30}$ Data were recorded using $1 \AA$ synchrotron radiation and a fixed glancing incidence angle of $0.5^{\circ}$, well above the critical angle of $0.20^{\circ}$ for total reflection at this energy. Two independent sets of data of two different samples were taken for both the cold and the hot conditions, to confirm reproducibility.

The cold surface showed both crystal truncation rods (CTRs) at integral $(h, k)$ positions in reciprocal space and superstructure rods (SSRs) at half-integral-half-integral positions $[h=m / 2, k=n / 2 ; m, n$ odd] and half-integral-integral positions $[h=m / 2, k=n / 2 ; m$ odd/even, $n$ even/odd]. It became clear during the structure refinement that the latter reflections could not be exclusively associated with a $(2 \times 2)$ reconstruction, and that a $(2 \times 1)$ reconstruction also had to be invoked. Both the CTR and SSR signals were stable under UHV conditions over the measurement time of over $24 \mathrm{~h}$.

The cold data set consists of 9 CTRs and 18 SSRs, comprising a total of 1668 nonequivalent structure factors. In addition, we recorded 806 equivalent data points in order to determine systematic differences between symmetryequivalent structure factors. Indeed, the total error is primarily associated with such systematic differences, most likely due to (a) the known imperfections of Verneuil-grown STO crystals $^{31}$ and (b) a slight bowing of the surface caused by the clamping mechanism to the heater. The error was determined to be $11.7 \%$, and symmetry-equivalent structure factors were averaged. The data set spans $|h|,|k|$, and $l=0$ to 3 $\left(Q \leqslant 8.36 \AA^{-1}\right)$.

The SXRD data of the hot sample was taken under typical conditions for thin film growth of perovskites, i.e., the STO was heated to $750 \pm 30^{\circ} \mathrm{C}$ in $10^{-3} \mathrm{~Pa} \mathrm{O}_{2}$. The SSRs gradually weakened during the heating period of about $30 \mathrm{~min}$., with only CTRs remaining. The SSRs did not return upon subsequent rapid cooling to cold conditions, indicating either an irreversible change in the surface structure or a kinetically hindered transition.

A set of 837 nonequivalent structure factors in 9 CTRs plus 764 equivalent data points was taken under the hot conditions. The systematic error between symmetry-equivalent structure factors was, at $28.9 \%$, significantly higher than for the cold data, and is probably attributable to mechanical distortions produced by thermal strain due to the heater/ clamping mechanism. The hot data set also spans reciprocal space for $|h|,|k|$, and $l=0$ to 3 .

In order to record nonspecular CTRs (and SSRs) in the stationary geometry, i.e., with a detector acceptance that is sufficiently large to integrate the complete diffraction signal over the entire in-plane direction in one single exposure, one can work out the minimum outgoing angle $\beta_{\text {out,min }}$, and (with respect to the STO lattice) the minimum $l_{\min }$ of a rod, above which independent structure factor amplitudes for a given sampling resolution $\Delta l$ are possible. ${ }^{32}$ Using an area pixel detector, this limit depends on the quality of the crystal and the energy resolution of the diffractometer. In the case of STO, typical in-plane rocking curves had widths of $\Delta Q_{\|}$ $=0.0044 \AA^{-1}$ (i.e., $\Delta \omega=0.02^{\circ}$ ). We thus obtain $l_{\min }=0.90$ and $l_{\min }=0.48$ for the sampling resolution used of $\Delta l=0.025$ and $\Delta l=0.05$ for CTRs and SSRs, respectively. This means that the diffraction data in the case of the CTRs are not completely resolved in the low- $l$ region. If we assume for this low- $l$ region that only half of the data points are reliable for the CTRs (but all for the SSRs, in agreement with the used sampling resolution), we get 1566 and 727 independent structure factor amplitudes for the cold and the hot data sets, respectively.

\section{B. Theoretical}

\section{Structure refinement}

Both the cold and the hot surface structures were determined using the program FIT. ${ }^{33}$ To refine parameters, it uses a robust grid search algorithm. A serious problem in all simple fitting procedures is the possibility of getting trapped in a local minimum. We tried to overcome this obstacle by iterative cycles of resetting fit parameters and refining the structure. For the presented surface structures, typically 50 iterations for both cold and hot STO were needed to obtain a final fit.

Domains were added incoherently. The sharp diffraction signals indicate large domain sizes, while the longitudinal coherence length of the focussed beam is $40 \mathrm{~nm}$ at a beam energy of $14 \mathrm{keV}$ for our source. ${ }^{28}$ Equivalent domains were equally weighted with regard to the surface symmetries, i.e., no a priori preferential orientation of the domains was assumed.

The final goodness-of-fit (GOF) is given in terms of the crystallographic $R$ factor, ${ }^{34,35}$ although optimization was carried out via $\chi^{2}$ minimization, in order to avoid artificial weighting of the most intense signal near the Bragg maxima in the CTRs. To be able to compare different models $\chi_{r}^{2}$, i.e., the reduced values, ${ }^{36}$ are always given. It is noted that, for the final model, changing from $\chi^{2}$ to minimization of the $R$ factor and back led to the same final model, within a reasonable set and range of reset fit parameters.

For the structures presented in this work, atomic coordinates, Debye-Waller (DW) factors, a general scaling factor, and, for the cold surface, fractional contributions from each surface-structure type were refined. Atomic movements are therefore represented by up to three parameters per site, depending on the surface symmetry assumed. The underlying bulk atoms had one isotropic DW factor for each $\mathrm{Sr}$, Ti, and O. In each of the lower ALs, isotropic DW factors were used for any particular atom type. Each atom of the top four ALs (or five in the DL surface terminations) was modeled using an anisotropic DW factor, with separate in- and out-of-plane components. As lower limits of the DW factors, $50 \%$ of the bulk values for $\mathrm{Sr}, \mathrm{Ti}$, and $\mathrm{O}$ were chosen. FIT uses DW factors in terms of $B$ values, according to international standards. ${ }^{37}$ For convenience, the DW factors presented here are converted into root mean squared displacements (RMSDs), i.e., the uncertainties of the atomic position due to thermal effects are given as the amplitudes of Gaussiandistributed oscillations, represented in units of the STO unit cell (see Tables II-V). Note that the DW factor accounts for both the dynamic motion and the static disorder with Gauss- 
ian distribution and may be called the "atomic displacement parameter" to stress explicitly the static component. ${ }^{38}$ Unless otherwise stated, every atom had an occupation parameter of unity. Note, however, that a high DW factor may also imply partial occupation. To model the two different surfaces with sets of parameters as described above, we used 394 parameters for the 112 atoms of the cold and 57 for 16 sites of the hot data, implying an oversampling of the independent structure factors by a factor of 4 and more than 10, respectively, i.e., well above the Nyquist criterion.

\section{Density functional theory calculations}

Density functional (DFT) calculations were performed using periodic slab models with a periodicity normal to the surface of $100 a_{0}$ units. This results in a slab separation of about $25 \AA$ for the thickest slabs, and more for thinner slabs. The slabs have a mirror symmetry (possibly combined with glide or inversion symmetry), resulting in a vanishing dipole moment across the slab. DFT equations were solved using the $\mathrm{DMOL}^{3}$ program, using default settings as described in Refs. 39-41.

The reciprocal space integrations were performed by sampling the Brillouin zone with unshifted $4 \times 4,2 \times 4$, and $2 \times 2$ meshes for the $(1 \times 1),(2 \times 1)$, and $(2 \times 2)$ surface unit cells, respectively. To estimate the sensitivity with respect to the density functional approximation, two sets of calculations were carried out, one with a local density functional Perdew-Wang-correlation (PWC), ${ }^{42}$ and the other including gradient corrections according to Perdew, Burke, and Ernzerhof (PBE) ${ }^{43}$

Wetting the surfaces by a high dielectric liquid, such as water, may change the relative surface energies. A simple estimate of the wetting effect is obtained by the conductorlike screening model applied to a surface (later referred to as "e-wet"). In this model, dielectric screening charges (i.e., mimmicing a conductorlike charge distribution) are semiempirically applied to the surface in order to simulate the effects of contact with water. ${ }^{44}$ Removing the $\mathrm{Sr}$ and its complexes by etching is also our rationale for comparing surfaces of different stoichiometry at low $\mathrm{Sr}$ chemical potential.

\section{Direct methods}

The challenge in diffraction based structure determination is the fact that the measured diffraction intensities are proportional to the squared amplitudes of the complex structure factors, but contain no information about their phases (the so-called "phase problem"). Therefore, a direct inversion of the Fourier components to retrieve the electron density in the crystal is impossible.

Conventional fit optimization programs such as FIT and ROD (Refs. 33 and 45) rely on detailed a priori knowledge of the system (i.e., the starting model) to overcome this problem. However, the probability of convergence to the correct solution depends very strongly on how well the initial model approximates the true structure. This problem becomes progressively severe with increased system complexity.

Although the missing phase data cannot be measured directly, 2D periodic structures such as crystal surfaces, in con- trast to 3D systems (i.e., bulk crystals), may provide this information by other means. Bulk diffraction data do not allow one to measure at least twice as many independent structure factors to reliably determine every single refinement parameter, as demanded by the Nyquist criterion, since only a discrete set of nonzero Fourier amplitudes, the Bragg peaks, can be determined. In surface diffraction data, however, the continuous signal along a CTR provides the necessary redundant information ("oversampling") needed for the application of so-called direct-or phase retrieval methods.

Several direct method attempts to solve the phase problem in surface crystallography have been reported. ${ }^{46-49}$ We used the PARADIGM method proposed by Saldin et al. to study the surface of hot STO. The algorithm aims to recover the surface structure by an iterative algorithm ${ }^{50}$ that alternately satisfies constraints in real and reciprocal space and exploits the fact that scattering from the unknown surface structure may be regarded as a perturbation of that from the truncated bulk structure.

\section{RESULTS AND DISCUSSION}

\section{A. Room-temperature structure}

\section{Starting models}

The presence of SSRs at half-integral-half-integral positions in $k$ space can in principle be explained by a $(2 \times 2)$ reconstruction completely covering the surface. However, as mentioned above, structural modeling showed that more than one reconstruction was required. A weighted mixture of a $(1 \times 1)$ relaxation and $(2 \times 1)$ and $(2 \times 2)$ reconstructions best reproduced the data.

In the following, we discuss the starting models for the three terminations that were combined to model the cold surface. Coordinates are given in units of the bulk STO cell, with the origin in the lower left corner.

(1) $(1 \times 1)$ models. Figure 3 summarizes the cold $(1 \times 1)$ models. The bulk STO unit cell terminates with an AL of $\mathrm{TiO}_{2}$ (a) placed on top of an $\mathrm{AL}$ of $\mathrm{SrO}$ (b). For the Ti-rich DL structures, either an $\mathrm{AL}$ of $\mathrm{TiO}_{2}$ (c) or TiO (d) was added on top of the bulk $\mathrm{TiO}_{2}$ layer (a). The structure in (e) is $\mathrm{O}$ deficient and was proposed to be energetically favorable compared to (a).$^{51}$ Another rowlike structure that was considered is produced by moving the $\mathrm{O}$ in (a) on top of the $\mathrm{Ti}$ atom (model f), instead of removing it (as in model e). To test the idea of Sr-enriched surfaces due to segregation during annealing suggested in Ref. 27, we added another $\mathrm{SrO}$ AL onto the Ti-rich DL structure shown in (c), either with the $\mathrm{Sr}$ in the $(1 / 21 / 2)$ position $(\mathrm{g})$ or at $(01 / 2)(\mathrm{h})$.

(2) $(2 \times 1)$ models. The $(2 \times 1)$ starting models are shown in Fig. 4. The AL in Fig. 4(a) sits on top of a bulk $\mathrm{TiO}_{2}$ layer, and together they form a $\mathrm{TiO}_{2}$-rich surface, as proposed by Erdman et al. ${ }^{14}$ Note that the Ti atoms form a characteristic zigzag motif. Two modifications of the top $\mathrm{TiO}_{2}$ layer of bulk STO were discussed by Castell ${ }^{15}$ in a scanning tunneling microscopy study (STM): (b) is a $\mathrm{TiO}_{2}$-depleted structure, in which only one $\mathrm{TiO}_{2}$-unit is present in a $(2 \times 1)$ reconstructed cell, whereas in (c) one $\mathrm{O}$ is removed to form a reconstruction with $\mathrm{Ti}_{2} \mathrm{O}_{3}$ stoichiometry. In (d), the Ti at- 

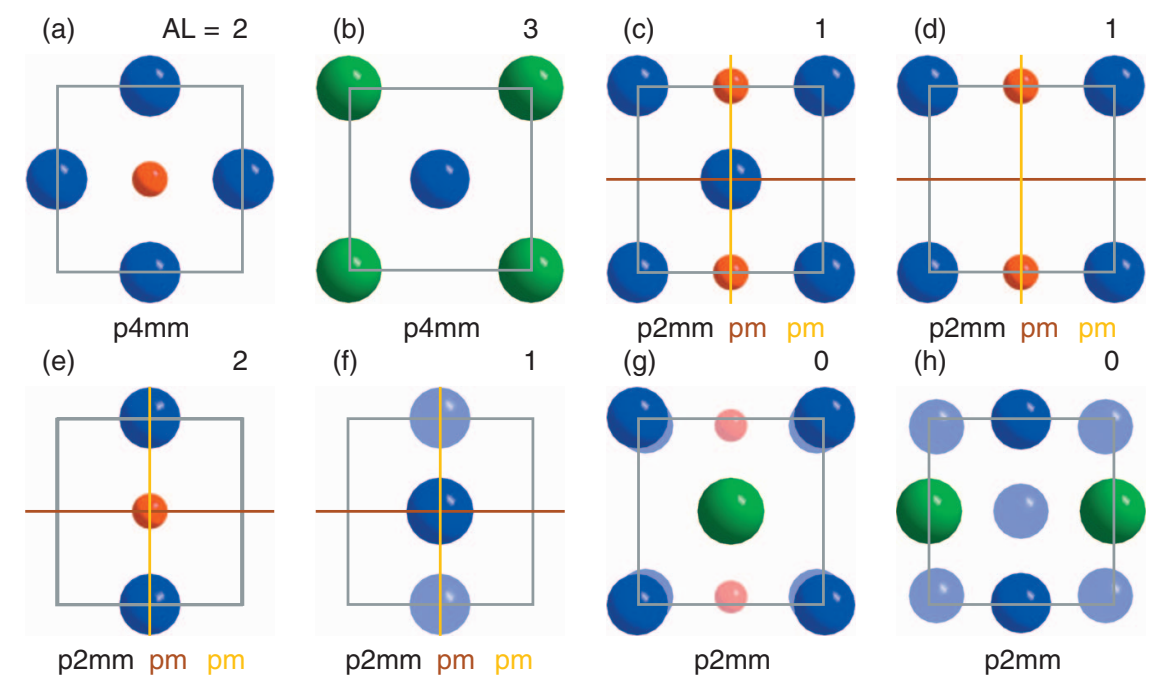

FIG. 3. (Color) Cold $(1 \times 1)$ terminations that are placed on STO bulk unit cells. The first two ALs of an ideal Ti-terminated bulk STO surface are shown in (a) and (b). All models are viewed from above onto the $a b$ plane and display the unit cell as a grey box, with the Sr bulk position at the cell corners. The number in the upper right corner indicates the AL of the opaque sites, labeling the surface layer of DL models as 1 . The adopted surface symmetries are noted in black below the cells where they are unambiguous. Other considered symmetry elements and their descriptions are highlighted in brown and yellow. Atoms shown in lighter shades represent nominal positions that are one AL deeper down, but are required to unambiguously understand the termination. A detailed description of the structures is given in the text. Color code: Sr green, Ti red, and O blue.

oms also form a zigzag motif, but in contrast to (a), this is a modification of the top $\mathrm{TiO}_{2}$ layer of bulk STO and is not a DL structure. The models in (e) and (f) are again $\mathrm{O}$ deficient, forming, respectively, a rowlike structure (as suggested by Noguera ${ }^{51}$, or a structure in which an $\mathrm{O}$ atom is moved on top of the adjacent Ti atom, similar to the cold $(1 \times 1)$ structure in Fig. 3(f). Assuming that an $\mathrm{O}$ atom moves with equal probability from the bulk position to the left or the right $\mathrm{Ti}$ atom, the occupation of the top $\mathrm{O}$ was set to 0.5 in model (f). Model (g) contains a Sr adatom layer with one $\mathrm{Sr}$ atom per $(2 \times 1)$ cell, as proposed in a STM study by Kubo and Nozoye. ${ }^{12}$ We note that the stability of $\mathrm{Sr}$ adatom surfaces has been questioned for high $\mathrm{Sr}$ coverages and could only be explained by first-principle calculations if the surface is far from equilibrium. ${ }^{20}$ Finally, models (h) and (i) have the same basic idea as presented in Figs. 3(g) and 3(h), respectively, i.e., an additional SrO layer on a Ti-rich DL structure.

(3) $(2 \times 2)$ models. The $(2 \times 2)$ reconstructions are shown in Fig. 5. Structures (a)-(c) all represent Ti-rich DLs on bulk $\mathrm{TiO}_{2}$ layers. In model (a), the fully stoichiometric $\mathrm{TiO}_{2}$ top layer consists of the characteristic zigzag motif and was found to be energetically favorable by DFT calculations presented by Warschkow et al. ${ }^{52}$ Note that this reconstruction is very similar to the $(2 \times 1)$ structure in Fig. $4(a)$ : it can be (a)

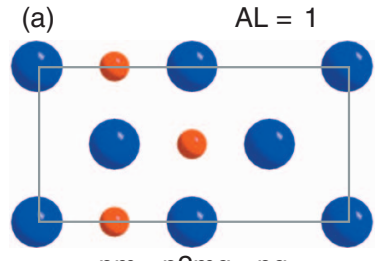

(d)
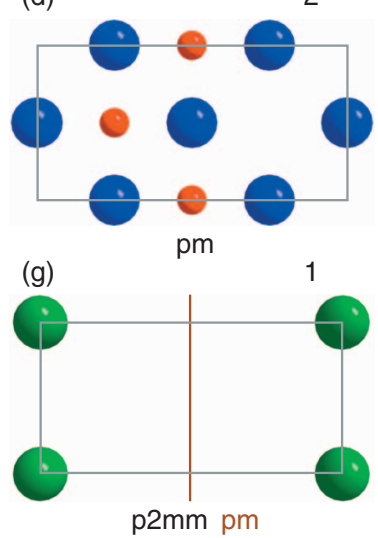

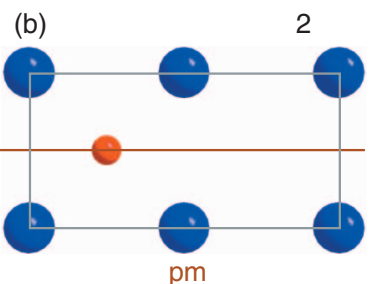

(e)

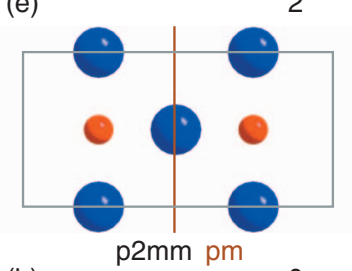

(h)

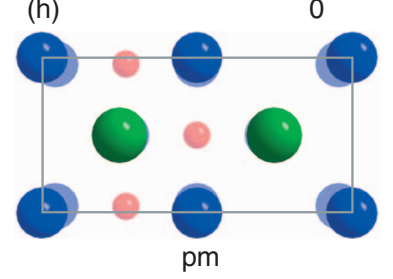

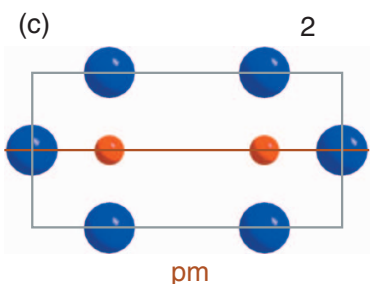

(f)
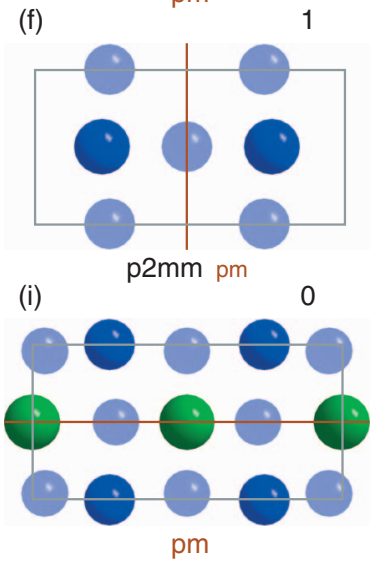

FIG. 4. (Color) The tested starting models for the cold $(2 \times 1)$ structures, using the same representation as in Fig. 3. 


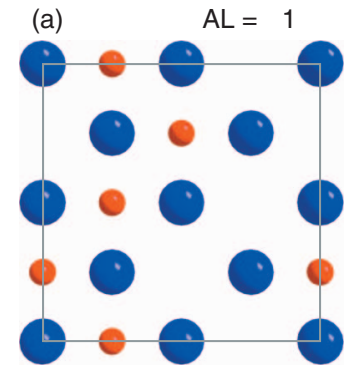

pm p2mg pg

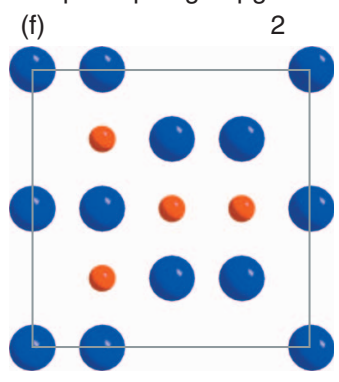

pm

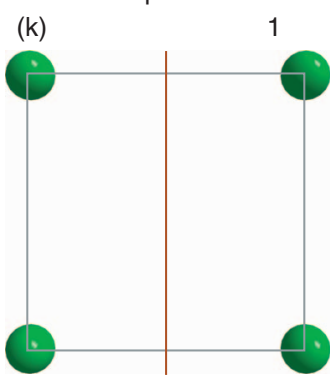

$\mathrm{p} 2 \mathrm{~mm}$

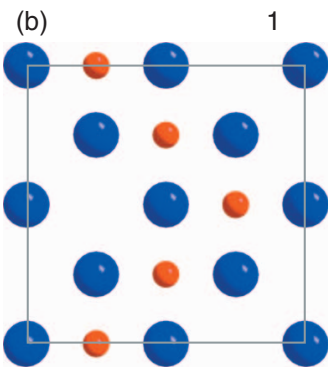

pm

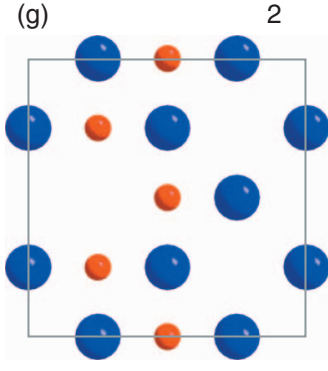

pm

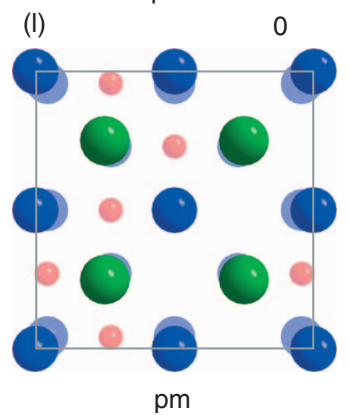

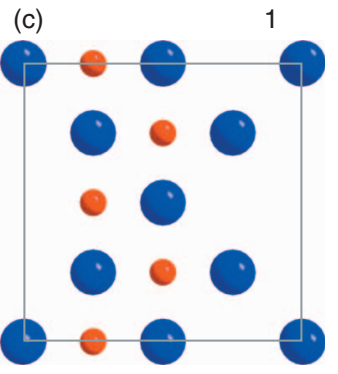

pm

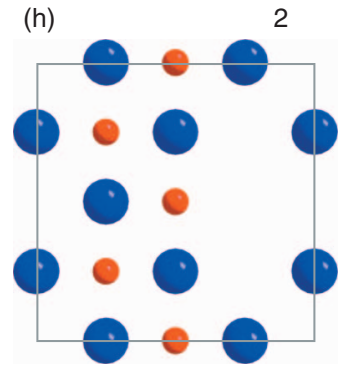

pm

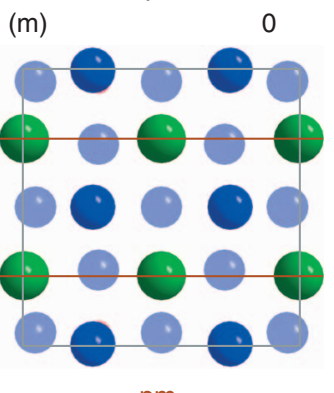

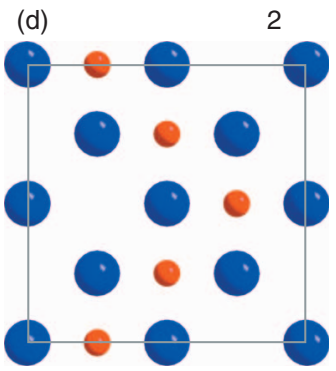

pm

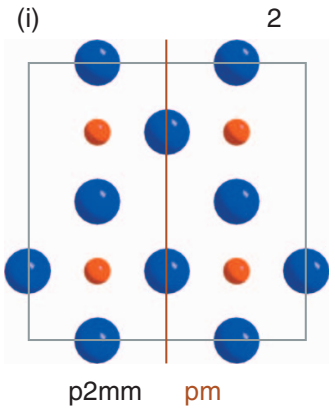

p2mm pm

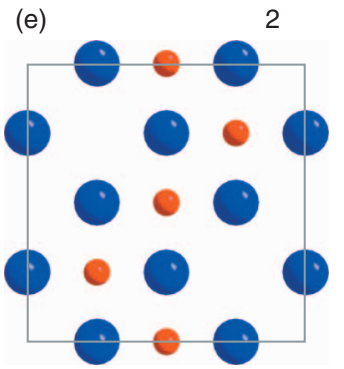

pg

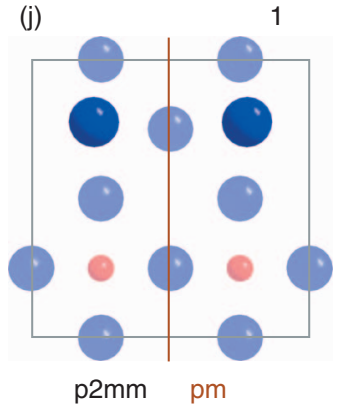

FIG. 5. (Color) Starting models for the cold $(2 \times 2)$ structures. Notation is the same as for Fig. 3 .

thought of as consisting of a juxtaposition of such a $(2 \times 1)$ structure and its mirror image. Structure (b) is very similar to (a), whereby the Ti atom zigzag motif is shifted by $(1 / 21 / 2)$ of a unit cell. Structure (c) consists of two adjacent $(2 \times 1)$ reconstructions of the type shown in Fig. 4(a), but due to $\mathrm{O}$ deficiency at the $\left(\begin{array}{ll}0 & 1\end{array}\right)$ position, it forms a true $(2 \times 2)$ reconstruction. The structures (d)-(h) are also based on Ti atom rearrangements, but in contrast to (a)-(c), no double Ti-layer is present, i.e., the changes take place in the top $\mathrm{TiO}_{2}$ layer of bulk STO. Model (d) looks thus the same as (b). The motif in (e) is similar to that in (a), but with the important differences of having two Ti sites on bulk Sr positions and the other two Ti atoms directly above bulk Ti. Model (f) shows an unusual $\mathrm{Ti}$ and $\mathrm{O}$ arrangement that would be difficult to explain by electrostatic arguments, but is presented for the sake of completeness. The two structures (g) and (h) are also based on the idea of two adjacent $(2 \times 1)$ reconstructions (with the $\mathrm{Ti}$ on top of bulk $\mathrm{Sr} / \mathrm{Ti}$ again) and $\mathrm{O}$ deficiencies [missing either the $(1 / 21)$ or the $(3 / 21)$ site] forming the $(2 \times 2)$ structures. For the models (i) and (j), we were inspired by an $\mathrm{O}$ deficiency (i) or an $\mathrm{O}$ movement on top of a bulk Ti site $(\mathrm{j})$, analogous to structures already presented for the $(1 \times 1)$ and $(2 \times 1)$ models. The same is true for the $\mathrm{Sr}$ adatom model $^{12}(\mathrm{k})$, or $\mathrm{SrO}$ overlayers on a $\mathrm{TiO}_{2}$-rich DL structure to form the structures (l) and (m), model (l) having varying $\mathrm{Sr}$ and $\mathrm{O}$ occupation (see Table I).

\section{Refinement}

The models presented in Sec. III A 1 were combined and fit to the experimental data. Table I shows the most promising 49 combinations. We note that, in assessing the potential of a model, we not only considered the $\chi_{r}^{2}$ or crystallographic $R$ factor, but also its physical reasonableness. These included sensible atom positions and distances between different ALs, or plausible DW factors. The fit procedure used a GOF criterion that was a weighted combination of the $\chi_{r}^{2}$ and a punitive energy term proportional to the square of the deviation from equilibrium bond lengths, similar to the "Keating energy" used in covalent structures. ${ }^{53}$ This second term helped to stabilize the models during the first few iterations. In general, we started to fit the top layers only, then subsequently added deeper ALs as the model relaxed. The weighting of the punitive energy term could then be reduced and the refinement iterated.

In arriving at the final model (number 36 in Table I), we were guided by a clear and consistent trend: all models containing a $\mathrm{TiO}_{2}$ DL and a zigzag motif of the top Ti atoms produced significantly better fits than those without these features. In addition, these models had sensible atomic positions and DW factors throughout.

The $(2 \times 1)$ and $(2 \times 2)$ reconstructions in models $1-6$ have a higher symmetry than other models in Table I. The resulting GOF values ( $\chi_{r}^{2}$ and $R$ factor) are high compared to 
TABLE I. Selected surface structure combinations under identical fit conditions. The letters in column $(1 \times 1),(2 \times 1)$, and $(2 \times 2)$ correspond to those shown in Figs. 3-5, respectively. Subscripted $h$ and $v$ depict horizontal and vertical mirror planes, respectively. Column $\mathrm{AL}$ gives the number of fit atomic layers, the last three columns are the fractional contributions of the terminations.

\begin{tabular}{|c|c|c|c|c|c|c|c|c|c|c|c|c|}
\hline Model & $(1 \times 1)$ & $(2 \times 1)$ & $(2 \times 2)$ & & Surface symm & tries & ALs & $\chi_{r}^{2}\left(|F|^{2}\right)$ & $R(|F|)$ & $f(1 \times 1)$ & $f(2 \times 1)$ & $f(2 \times 2)$ \\
\hline 1 & $a$ & $e$ & $i$ & $p 4 m m$ & $p 2 m m$ & $p 2 m m$ & 4 & 4.416 & 0.154 & 0.150 & 0.316 & 0.534 \\
\hline 2 & $a$ & $f$ & $j$ & $\mathrm{p} 4 \mathrm{~mm}$ & $\mathrm{p} 2 \mathrm{~mm}$ & $\mathrm{p} 2 \mathrm{~mm}$ & 4 & 6.218 & 0.166 & 0.188 & 0.307 & 0.505 \\
\hline 3 & $f$ & $f$ & $j$ & $\mathrm{p} 2 \mathrm{~mm}$ & $\mathrm{p} 2 \mathrm{~mm}$ & $\mathrm{p} 2 \mathrm{~mm}$ & 4 & 5.686 & 0.164 & 0.108 & 0.312 & 0.580 \\
\hline 4 & $a$ & $g$ & $k$ & $p 4 m m$ & $\mathrm{p} 2 \mathrm{~mm}$ & $\mathrm{p} 2 \mathrm{~mm}$ & 5 & 3.745 & 0.140 & 0.341 & 0.328 & 0.331 \\
\hline 5 & $c$ & & $a$ & $\mathrm{p} 2 \mathrm{~mm}$ & & $p 2 m g$ & 5 & 5.146 & 0.146 & 0.437 & & 0.563 \\
\hline 6 & $c$ & $a$ & $a$ & $\mathrm{p} 2 \mathrm{~mm}$ & $p 2 m g$ & $p 2 m g$ & 5 & 2.903 & 0.131 & 0.361 & 0.180 & 0.459 \\
\hline 7 & $a$ & $f$ & $j$ & $p 4 m m$ & $p m_{v}$ & $p m_{v}$ & 4 & 2.556 & 0.144 & 0.214 & 0.359 & 0.427 \\
\hline 8 & $f$ & $f$ & $j$ & $p m_{v}$ & $p m_{v}$ & $\mathrm{pm}_{v}$ & 4 & 2.647 & 0.135 & 0.167 & 0.411 & 0.422 \\
\hline 9 & $a$ & $e$ & $i$ & $p 4 m m$ & $p m_{v}$ & $p m_{v}$ & 4 & 3.025 & 0.140 & 0.135 & 0.377 & 0.488 \\
\hline 10 & $a$ & $b$ & $e$ & $p 4 m m$ & $\mathrm{pm}_{h}$ & $p g$ & 4 & 3.058 & 0.134 & 0.000 & 0.501 & 0.499 \\
\hline 11 & $a$ & $b$ & $d$ & $p 4 m m$ & $\mathrm{pm}_{h}$ & $p m_{h}$ & 4 & 2.228 & 0.133 & 0.238 & 0.340 & 0.422 \\
\hline 12 & $a$ & $b$ & $f$ & $p 4 m m$ & $p m_{h}$ & $p m_{h}$ & 4 & 2.313 & 0.127 & 0.172 & 0.352 & 0.476 \\
\hline 13 & $a$ & $b$ & $b$ & $p 4 m m$ & $\mathrm{pm}_{h}$ & $\mathrm{pm}_{h}$ & 4 & 2.282 & 0.131 & 0.203 & 0.329 & 0.468 \\
\hline 14 & $a$ & $c$ & $e$ & $p 4 m m$ & $\mathrm{pm}_{h}$ & $p g$ & 4 & 2.571 & 0.132 & 0.214 & 0.418 & 0.368 \\
\hline 15 & $a$ & $c$ & $d$ & $\mathrm{p} 4 \mathrm{~mm}$ & $\mathrm{pm}_{h}$ & $\mathrm{pm}_{h}$ & 4 & 2.287 & 0.132 & 0.145 & 0.391 & 0.464 \\
\hline 16 & $a$ & $c$ & $f$ & $p 4 m m$ & $\mathrm{pm}_{h}$ & $\mathrm{pm}_{h}$ & 4 & 2.759 & 0.137 & 0.000 & 0.468 & 0.532 \\
\hline 17 & $a$ & $c$ & $b$ & $p 4 m m$ & $p m_{h}$ & $p m_{h}$ & 4 & 2.328 & 0.128 & 0.145 & 0.416 & 0.439 \\
\hline 18 & $a$ & $a$ & $e$ & $\mathrm{p} 4 \mathrm{~mm}$ & $\mathrm{pm}_{h}$ & $p g$ & 4 & 2.909 & 0.123 & 0.169 & 0.447 & 0.384 \\
\hline 19 & $a$ & $a$ & $d$ & $p 4 m m$ & $\mathrm{pm}_{h}$ & $\mathrm{pm}_{h}$ & 4 & 2.169 & 0.122 & 0.252 & 0.470 & 0.278 \\
\hline 20 & $a$ & $a$ & $f$ & $\mathrm{p} 4 \mathrm{~mm}$ & $\mathrm{pm}_{h}$ & $\mathrm{pm}_{h}$ & 4 & 2.351 & 0.115 & 0.187 & 0.468 & 0.345 \\
\hline 21 & $a$ & $d$ & $g$ & $p 4 m m$ & $\mathrm{pm}_{h}$ & $\mathrm{pm}_{h}$ & 4 & 2.129 & 0.111 & 0.297 & 0.380 & 0.323 \\
\hline 22 & $a$ & $d$ & $h$ & $p 4 m m$ & $p m_{h}$ & $p m_{h}$ & 4 & 2.183 & 0.118 & 0.256 & 0.341 & 0.403 \\
\hline 23 & $a$ & & $b$ & $p 4 m m$ & & $\mathrm{pm}_{h}$ & 5 & 3.468 & 0.135 & 0.385 & & 0.615 \\
\hline 24 & $a$ & $g$ & $k$ & $p 4 m m$ & $p m_{v}$ & $p m_{v}$ & 5 & 2.624 & 0.132 & 0.263 & 0.345 & 0.392 \\
\hline 25 & $a$ & $a$ & $b$ & $\mathrm{p} 4 \mathrm{~mm}$ & $\mathrm{pm}_{h}$ & $p m_{h}$ & 5 & 2.069 & 0.118 & 0.185 & 0.386 & 0.429 \\
\hline 26 & & $a$ & $b$ & & $p m_{h}$ & $p m_{h}$ & 5 & 2.211 & 0.120 & & 0.516 & 0.484 \\
\hline 27 & $c$ & $a$ & $b$ & $p 2 \mathrm{~mm}$ & $\mathrm{pm}_{h}$ & $\mathrm{pm}_{h}$ & 5 & 1.978 & 0.114 & 0.218 & 0.422 & 0.360 \\
\hline 28 & $c$ & $a$ & $b$ & $p m_{h}$ & $\mathrm{pm}_{h}$ & $p m_{h}$ & 5 & 2.059 & 0.113 & 0.225 & 0.383 & 0.392 \\
\hline 29 & $c$ & $a$ & $b$ & $p m_{v}$ & $p m_{h}$ & $p m_{h}$ & 5 & 1.869 & 0.113 & 0.208 & 0.421 & 0.371 \\
\hline 30 & $d$ & $a$ & $b$ & $p 2 \mathrm{~mm}$ & $\mathrm{pm}_{h}$ & $\mathrm{pm}_{h}$ & 5 & 2.172 & 0.119 & 0.170 & 0.422 & 0.408 \\
\hline 31 & $d$ & $a$ & $b$ & $p m_{h}$ & $\mathrm{pm}_{h}$ & $\mathrm{pm}_{h}$ & 5 & 2.190 & 0.113 & 0.173 & 0.378 & 0.449 \\
\hline 32 & $d$ & $a$ & $b$ & $\mathrm{pm}_{v}$ & $\mathrm{pm}_{h}$ & $\mathrm{pm}_{h}$ & 5 & 1.878 & 0.112 & 0.217 & 0.431 & 0.352 \\
\hline 33 & $c$ & $a^{\mathrm{a}}$ & $c$ & $p 2 m m$ & $\mathrm{pm}_{h}$ & $\mathrm{pm}_{h}$ & 5 & 1.771 & 0.114 & 0.272 & 0.341 & 0.387 \\
\hline 34 & $c$ & $a^{\mathrm{a}}$ & $c$ & $\mathrm{p} 2 \mathrm{~mm}$ & $\mathrm{pm}_{h}$ & $p m_{h}$ & 5 & 2.059 & 0.108 & 0.187 & 0.379 & 0.434 \\
\hline 35 & $c$ & $a$ & $c$ & $\mathrm{p} 2 \mathrm{~mm}$ & $\mathrm{pm}_{h}$ & $\mathrm{pm}_{h}$ & 5 & 1.713 & 0.116 & 0.298 & 0.353 & 0.349 \\
\hline 36 & $c$ & $a$ & $a$ & $\mathrm{p} 2 \mathrm{~mm}$ & $\mathrm{pm}_{h}$ & $\mathrm{pm}_{h}$ & 5 & 1.913 & 0.118 & 0.244 & 0.363 & 0.393 \\
\hline 37 & $c$ & $a$ & $a$ & $p 2 m m$ & $p m_{h}$ & $p 2 m g$ & 5 & 2.859 & 0.116 & 0.215 & 0.443 & 0.342 \\
\hline 38 & $c$ & $a$ & $a$ & $\mathrm{p} 2 \mathrm{~mm}$ & $\mathrm{pm}_{h}$ & $p g$ & 5 & 2.263 & 0.120 & 0.187 & 0.419 & 0.394 \\
\hline 39 & $c$ & $a^{\mathrm{b}}$ & $a^{\mathrm{b}}$ & $\mathrm{p} 2 \mathrm{~mm}$ & $p 2 m g / p m_{h}$ & $\mathrm{p} 2 \mathrm{mg} / \mathrm{pm}_{h}$ & 5 & 2.154 & 0.118 & 0.251 & 0.366 & 0.383 \\
\hline $40^{\mathrm{c}}$ & $c$ & $a^{\mathrm{b}}$ & $a^{\mathrm{b}}$ & $\mathrm{p} 2 \mathrm{~mm}$ & $\mathrm{pg} / \mathrm{pm}_{h}$ & $\mathrm{pg} / \mathrm{pm}_{h}$ & 5 & 1.784 & 0.116 & 0.190 & 0.401 & 0.409 \\
\hline $41^{\mathrm{c}}$ & $c$ & $a$ & $a^{\mathrm{b}}$ & $\mathrm{p} 2 \mathrm{~mm}$ & $\mathrm{pm}_{h}$ & $\mathrm{p} 2 \mathrm{mg} / \mathrm{pm}_{h}$ & 5 & 1.873 & 0.114 & 0.228 & 0.343 & 0.429 \\
\hline $42^{\mathrm{c}}$ & $c$ & $a$ & $a^{\mathrm{b}}$ & $\mathrm{p} 2 \mathrm{~mm}$ & $\mathrm{pm}_{h}$ & $\mathrm{pg} / \mathrm{pm}_{h}$ & 5 & 1.580 & 0.112 & 0.224 & 0.323 & 0.453 \\
\hline $43^{c}$ & $c$ & $a^{\mathrm{a}}$ & $a^{\mathrm{b}}$ & $\mathrm{p} 2 \mathrm{~mm}$ & $p m_{h}$ & $p g / p m_{h}$ & 5 & 1.676 & 0.120 & 0.168 & 0.404 & 0.428 \\
\hline $44^{c}$ & $c$ & $a^{\mathrm{b}}$ & $a^{\mathrm{b}}$ & $p 2 m m$ & $\mathrm{pm}_{h}$ & $p g / p 2 m g$ & 5 & 2.551 & 0.118 & 0.218 & 0.405 & 0.377 \\
\hline $45^{\mathrm{c}}$ & $g^{\mathrm{d}}$ & $h^{\mathrm{d}}$ & $l^{\mathrm{d}}$ & $p 2 m m$ & $p m_{h}$ & $p m_{h}$ & 6 & 1.506 & 0.105 & 0.342 & 0.352 & 0.306 \\
\hline $46^{\mathrm{c}}$ & $g^{\mathrm{d}}$ & $h^{\mathrm{d}}$ & $l^{\mathrm{d}}$ & $\mathrm{p} 2 \mathrm{~mm}$ & $\mathrm{pm}_{h}$ & $\mathrm{pm}_{h}$ & 5 & 1.393 & 0.102 & 0.181 & 0.265 & 0.554 \\
\hline $47^{\mathrm{c}}$ & $g^{\mathrm{e}}$ & $h^{\mathrm{d}}$ & $l^{\mathrm{d}}$ & $\mathrm{p} 2 \mathrm{~mm}$ & $\mathrm{pm}_{h}$ & $\mathrm{pm}_{h}$ & 6 & 1.265 & 0.103 & 0.292 & 0.299 & 0.409 \\
\hline $48^{c}$ & $g^{\mathrm{e}}$ & $h^{\mathrm{e}}$ & $l^{\mathrm{e}}$ & $\mathrm{p} 2 \mathrm{~mm}$ & $p m_{h}$ & $p m_{h}$ & 5 & 1.335 & 0.105 & 0.238 & 0.350 & 0.412 \\
\hline $49^{c}$ & $h$ & $i$ & $m$ & $p 2 m m$ & $p m_{h}$ & $p m_{h}$ & 6 & 1.971 & 0.136 & 0.000 & 0.427 & 0.573 \\
\hline
\end{tabular}

${ }^{\mathrm{a}} \mathrm{O}$ moved out of the plane, similar to Ref. 14.

${ }^{\mathrm{b}}$ Top layer has other symmetry than underneath.

${ }^{\mathrm{c}}$ Refined atomic positions unphysical.
${ }^{\mathrm{d}} \mathrm{Sr}$ and $\mathrm{O}$ occupation $=1.0$.

${ }^{\mathrm{e}} \mathrm{Sr}$ and $\mathrm{O}$ occupation $=0.5$. 
other models. This suggests that reconstructions with lower surface symmetries are preferred.

Models 7-25 all have a $\mathrm{TiO}_{2}$-terminated $(1 \times 1)$ structure without a double layer. All the $\chi_{r}^{2}$ values are higher than 2.1. Note that models with a Ti atom zigzag motif often display low $\chi_{r}^{2}$ and $R$ values within this group.

Models 27-44 contain Ti-rich DLs. The $\chi_{r}^{2}$ values are around 2 with $R$ factors of 0.120 or lower. Also, the fractional contributions of the different terminations [about $20 \%$ $(1 \times 1), 40 \%(2 \times 1)$, and $40 \%(2 \times 2)]$ vary less from model to model than for models $7-25$. The first set (27-36) are modeled with $p 2 \mathrm{~mm}$ and $\mathrm{pm}$ symmetries, whereas in the second set (37-44) glide mirror symmetries ( $p 2 m g$ and $p g$ ) are also used, as these belong naturally to the zigzag motif. If the glide symmetry acted on the entirety of the $(2 \times 2)$ domain, the fit was poorer and the models (37 and 38) were physically questionable. By introducing a physically dubious mixed-symmetry within either the $(2 \times 1)$ or $(2 \times 2)$ reconstructions (i.e., the top layer having glide symmetry and everything below, a mirror plane, models 39-43) the fits improved considerably. However, as can be seen in Table I, these models have been deemed to be unphysical. This somewhat subjective judgement was based on the fact that the majority of the atomic positions are unphysically disordered, although the shifts of the individual atoms are generally not unreasonable.

In contrast, our preferred model 36 , contains atom positions that appear to remain closer to the starting positions, especially below the top two or three AL. ${ }^{21}$ Although it does not have the lowest $\chi_{r}^{2}$ at this point of the refinement procedure, it was chosen for the following reasons. First, the model is supported by previous experimental evidence regarding the DL $(2 \times 1)$ domain, ${ }^{14}$ and also by DFT calculations, which predict the low surface energy of STO terminated with the DL $(2 \times 2)$ described here. ${ }^{52}$ Indeed, our own DFT calculations (see Sec. III C) have shown that the three lowest surface energy configurations include the $(2 \times 1)$ and $(2 \times 2)$ reconstructions of model 36 . The atomic positions as well as the DW factors (for the surface and the bulk) seemed to be most physically reasonable, compared to all other DL structures. In particular, the Ti atoms of the zigzag motif, which seems to be so important for producing a low surface energy, barely shift from their start positions.

The GOF of combinations containing a DL- $(1 \times 1)$ relaxation are better than those with a bulk $(1 \times 1)$ termination, but are nevertheless of comparable magnitude. At the point of the refinement procedure presented in Table I, adopting a $\mathrm{TiO}_{2}$-DL for the $(1 \times 1)$ termination does therefore not seem to be necessary. There are, however, pressing arguments to postulate such a DL structure. First, the combination of DL $(2 \times 1)$ and $(2 \times 2)$ reconstructions with a bulk $(1 \times 1)$ domain would imply half unit cell (i.e., $2 \AA$ ) height steps at their boundaries, of which we see no evidence in the AFM pictures (see Fig. 1). A second argument lies in the fact that the reconstructions vanish upon annealing. It seems unlikely that a mostly DL-reconstructed surface transforms to a bulklike terminated $(1 \times 1)$ when the substrate is brought to conditions typical for thin film growth, which would require the evaporation of the uppermost $\mathrm{TiO}_{2} \mathrm{AL}$ of the $(2 \times 1)$ and
$(2 \times 2)$ domains, despite the fact that DFT calculations predict that they should be the most stable configurations. Moreover, the existence of an "ideal" bulk $(1 \times 1)$ termination in UHV has been recently questioned, ${ }^{17}$ based on arguments of surface oxygen loss. Finally, the $\mathrm{TiO}_{2}$-DL $(1 \times 1)$ model fits the experimental data better than a bulk $(1 \times 1)$, when the number of fitted AL is increased.

Models 45-49 investigate the possibility of $\mathrm{SrO}$ segregation during the annealing process. ${ }^{27}$ The models consisting of such terminations (i.e., models 45-48), have $\chi_{r}^{2}$ values and $R$ factors that are clearly superior than those of any of the other presented models. This is, however, at the expense of physical reasonableness. The fitted structures all show the same trend: The top SrO layer is repelled significantly towards vacuum for all terminations, resulting in an interlayer distance of over $3 \AA$, instead of $2 \AA$. In addition, the side views of the structures appear to be considerably bent.

\section{Final structure}

The experimental data and the calculated intensities of model 36 are shown in Fig. 6. The intensity in the SSRs varies strongly with a periodicity of the order of $1 / 3$ of a reciprocal lattice unit. Thus, significant atomic displacements are expected down to three unit cells, which, indeed, we found in the refinement. The quality of the data and the fits is evidenced by a final $R$ factor of $4.5 \%$ and the absence of unphysical positions or DW factors. The structure of the final fit model is shown in Figs. 7(a)-7(c) ${ }^{54}$ The reconstructions dominate the surface termination with percentages of $43 \%$ of $(2 \times 2)$ (c) and $37 \%$ of $(2 \times 1)$ (b). A smaller contribution of $20 \%$ (a) could be attributed to a $(1 \times 1)$ relaxation. Analysis of four rods of a second STO substrate gave very similar intensities (within 5\%) and thus essentially identical weightings of the different reconstruction/relaxation domains. The content of impurities may play a role as well, as argued in Sec. III D.

The atomic coordinates of the $(1 \times 1),(2 \times 1)$, and $(2 \times 2)$ domains are listed in Tables II-IV, respectively. The atoms are grouped in ALs with a positive $z$ axis pointing out of the surface, and the $z=0$ position referring to that layer where the atoms are not shifted, i.e., they are on bulk positions. The first and the second ALs form the DL structure. The fourth column represents the high-symmetry starting position of the atom. The coordinates are given in fractions of bulk STO unit cells, with the associated DW factors as RMSDs. The atoms near the surface generally undergo more pronounced movements than do atoms deeper down and have larger DW factors. A large DW factor may reflect less than unit occupation for some of the atoms, although we have no evidence of vacancies from our fits. Note that for the $(1 \times 1)$ relaxation, no in-plane movements are allowed due to the $p 2 \mathrm{~mm}$ symmetry. A further discussion of the cold structure with regards to an order-disorder transition will be given in Sec. III E.

\section{B. Hot structure}

\section{Starting models}

The hot data set consisted of CTRs, with no SSRs present. Thus, we only considered the set of $(1 \times 1)$ terminations 

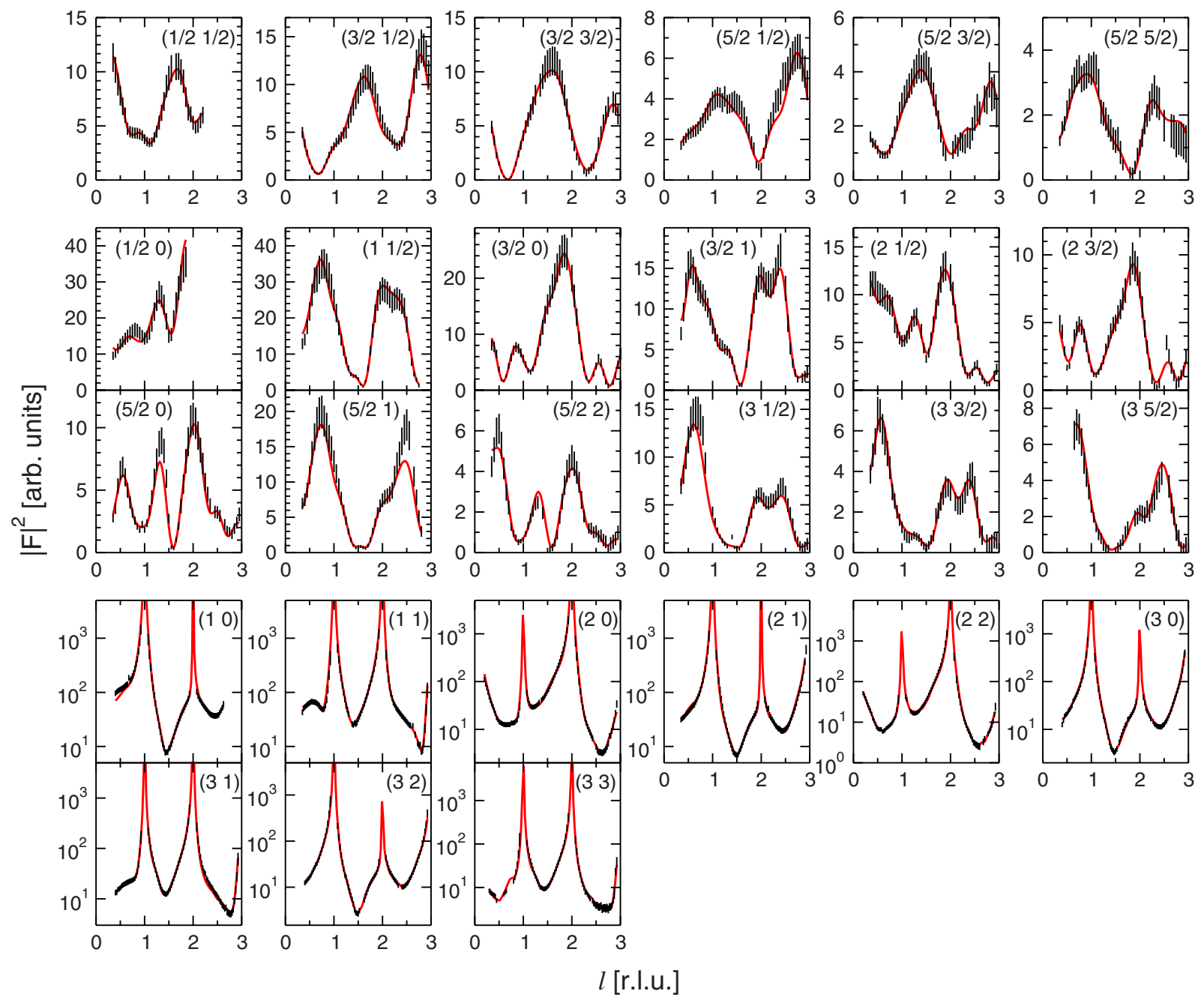

FIG. 6. (Color online) Set of the SXRD data (black data points) and calculated intensities (gray solid line, red online) for the cold conditions. The top row contains the six SSRs that can be exclusively associated with the $(2 \times 2)$ reconstruction. The central group of 12 SSRs can represent signal from both the $(2 \times 2)$ and $(2 \times 1)$ domains, and indeed we see that their intensities are about double those of the uppermost row, indicative that a $(2 \times 1)$ does exist. The nine CTRs at integer positions in $k$ space are presented on a logarithmic scale in the lowest group.

shown in Fig. 8. All these $(1 \times 1)$ structures contain double layers.

Figure 8(a) shows the top AL of the $\mathrm{TiO}_{2}$ DL structure that was also found in the cold data. The oxygen overlayer structure in (b) was recently suggested by Vonk et al., ${ }^{18}$ where they studied a chemically etched and thermally treated $(1 \times 1)$ STO $(001)$ surface via SXRD at room temperature and in air.

As for the reconstructed (cold) case, both $p 4 \mathrm{~mm}$ symmetry and multiple domains of $p 2 \mathrm{~mm}$ are consistent with the diffraction pattern; PARADIGM treats these cases separately. When $p 2 m m$ symmetry was assumed, PARADIGM suggested the same $\mathrm{TiO}_{2}$-DL surface as for the cold model (model a). This result will be discussed further in Sec. III B 3. When $p 4 \mathrm{~mm}$ symmetry was imposed, PARADIGM suggested four further starting models, all of which contain a metal-rich top surface layer, shown in models (c)-(f). The top AL either consists of $\mathrm{Sr}, \mathrm{Ti}$ or their mixture. Note that all surfaces suggested by PARADIGM contain a double layer. All of these models were subsequently tested using conventional structure refinement with fit, as described below.

\section{Refinement}

The different structural models for the hot data set were fit using identical refinement procedures. In Fig. 9, we present the evolution of the GOF criteria $\chi_{r}^{2}$ (a) and $R$ factor (b) as a function of the number of fit ALs. All the models were directly fit down to the shown number of ALs. The models are labeled corresponding to Fig. 8, plus a bulk model without a Ti-rich DL.

The best results are obtained when the $\mathrm{TiO}_{2}$-DL structure is fit to the data, both in terms of $\chi_{r}^{2}$ and the $R$ factor. The bulk and the $\mathrm{O}$ overlayer fit worse than the DL structure. In particular, the $\mathrm{O}$ overlayer model reaches unphysical positions for 3 and 4 ALs, i.e., the $\mathrm{O}$ atoms are compressed into the next underlying layer. Indeed, the shapes of the CTRs differ significantly between the SXRD data presented here and that from Vonk et al., ${ }^{18}$ which is perhaps not surprising, 
(a)

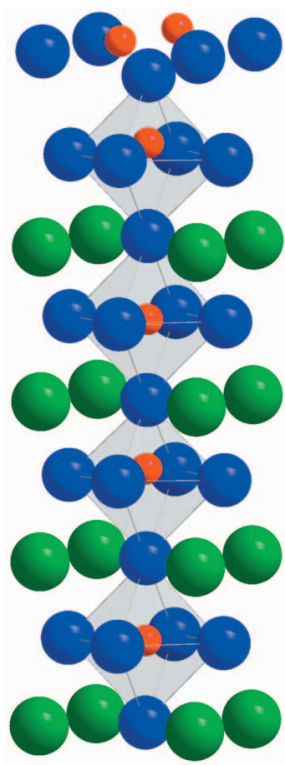

(1x1), p2mm, 20\% cold

(b)

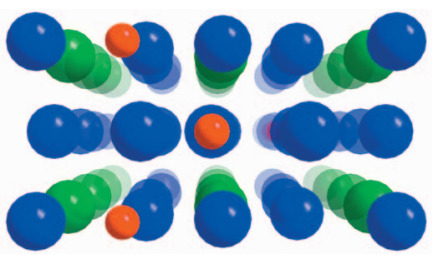

(2x1), pm, 37\%

(c)

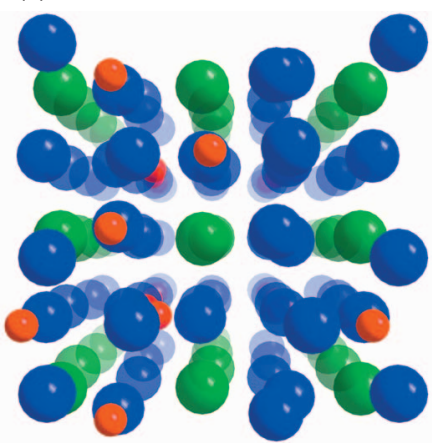

(2x2), pm, $43 \%$

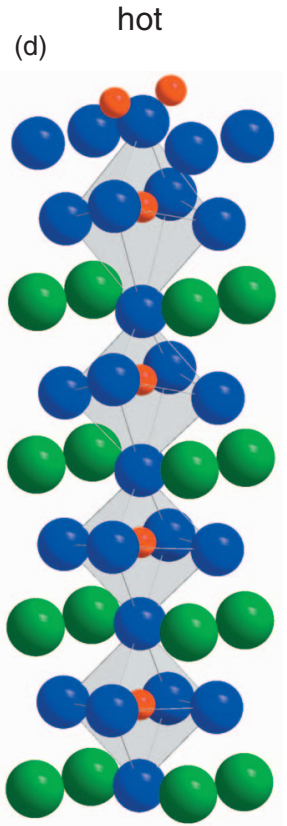

(1x1), p2mm
FIG. 7. (Color) The final models for the cold domains (a)-(c) and the hot structure (d), including their symmetries and percentage contributions. The $(1 \times 1)$ structures [cold (a) and hot (d)] are viewed from the side, while the reconstructions are from above. Color code: $\mathrm{Sr}$ green, Ti red, and O blue. given that their study was performed in air and at room temperature, whereas our data was taken under film growth conditions. We also note that we have observed several times that metal oxides samples in air exposed to glancingincidence synchrotron radiation show clear radiation damage within less than an hour. Also, from a chemical perspective, a dense packing of the surface with $\mathrm{O}$ is difficult to explain, either with hydrogen-bonded oxygen, where the experimentally found bonds are too short, or with covalent bonds, where they are too long. Moreover, it is rather unlikely for a

TABLE II. Refined positions and DW factors (expressed as root mean squared displacements in brackets) for the $(1 \times 1)$ reconstruction under cold conditions in units of bulk STO.

\begin{tabular}{|c|c|c|c|c|c|c|}
\hline $\mathrm{AL}$ & No. & Atom & $\begin{array}{l}\text { Nominal } \\
\text { position }\end{array}$ & $x / a_{0}$ & $y / a_{0}$ & $z / a_{0}$ \\
\hline \multirow[t]{3}{*}{1} & 1 & $\mathrm{Ti}$ & $\left(\begin{array}{lll}\frac{1}{2} & 0 & 3\end{array}\right)$ & $0.500(54)$ & $0.000(54)$ & $3.187(69)$ \\
\hline & 2 & $\mathrm{O}$ & $\left(\begin{array}{lll}0 & 0 & 3\end{array}\right)$ & $0.000(20)$ & $0.000(20)$ & $3.107(20)$ \\
\hline & 3 & $\mathrm{O}$ & $\left(\begin{array}{lll}\frac{1}{2} & \frac{1}{2} & 3\end{array}\right)$ & $0.500(118)$ & $0.500(118)$ & $2.940(57)$ \\
\hline \multirow[t]{3}{*}{2} & 4 & $\mathrm{Ti}$ & $\left(\begin{array}{lll}\frac{1}{2} & \frac{1}{2} & \frac{5}{2}\end{array}\right)$ & $0.500(14)$ & $0.500(14)$ & $2.529(34)$ \\
\hline & 5 & $\mathrm{O}$ & $\left(\begin{array}{lll}\frac{1}{2} & 0 & \frac{5}{2}\end{array}\right)$ & $0.500(25)$ & $0.000(25)$ & $2.457(204)$ \\
\hline & 6 & $\mathrm{O}$ & $\left(\begin{array}{lll}0 & \frac{1}{2} & \frac{5}{2}\end{array}\right)$ & $0.000(20)$ & $0.500(20)$ & $2.442(20)$ \\
\hline \multirow[t]{2}{*}{3} & 7 & $\mathrm{Sr}$ & $\left(\begin{array}{lll}0 & 0 & 2\end{array}\right)$ & $0.000(55)$ & $0.000(55)$ & $1.916(132)$ \\
\hline & 8 & $\mathrm{O}$ & 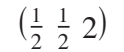 & $0.500(20)$ & $0.500(20)$ & $1.962(64)$ \\
\hline \multirow[t]{3}{*}{4} & 9 & $\mathrm{Ti}$ & $\left(\begin{array}{lll}\frac{1}{2} & \frac{1}{2} & \frac{3}{2}\end{array}\right)$ & $0.500(127)$ & $0.500(127)$ & $1.455(72)$ \\
\hline & 10 & $\mathrm{O}$ & $\left(\begin{array}{lll}\frac{1}{2} & 0 & \frac{3}{2}\end{array}\right)$ & $0.500(20)$ & $0.000(20)$ & $1.462(20)$ \\
\hline & 11 & $\mathrm{O}$ & $\left(0 \frac{1}{2} \frac{3}{2}\right)$ & $0.000(20)$ & $0.500(20)$ & $1.458(20)$ \\
\hline \multirow[t]{2}{*}{5} & 12 & $\mathrm{Sr}$ & $\left(\begin{array}{lll}0 & 0 & 1\end{array}\right)$ & $0.000(68)$ & $0.000(68)$ & $0.975(197)$ \\
\hline & 13 & $\mathrm{O}$ & $\left(\begin{array}{lll}\frac{1}{2} & \frac{1}{2} & 1\end{array}\right)$ & $0.500(78)$ & $0.500(78)$ & $0.964(61)$ \\
\hline \multirow[t]{3}{*}{6} & 14 & $\mathrm{Ti}$ & $\left(\begin{array}{lll}\frac{1}{2} & \frac{1}{2} & \frac{1}{2}\end{array}\right)$ & $0.500(44)$ & $0.500(44)$ & $0.546(44)$ \\
\hline & 15 & $\mathrm{O}$ & $\left(\begin{array}{lll}\frac{1}{2} & 0 & \frac{1}{2}\end{array}\right)$ & $0.500(20)$ & $0.000(20)$ & $0.505(20)$ \\
\hline & 16 & $\mathrm{O}$ & $\left(\begin{array}{lll}0 & \frac{1}{2} & \frac{1}{2}\end{array}\right)$ & $0.000(20)$ & $0.500(20)$ & $0.492(20)$ \\
\hline
\end{tabular}

perovskite system to have mostly ionic bonds in the bulk and a covalent nature at the surface.

The bulk model consistently produces marginally poorer results than the $\mathrm{TiO}_{2}$-DL model. Both models give similar results when only $1 \mathrm{AL}$ was fit, but successive layers improve the quality of the fit for the DL structure compared to bulk (see Fig. 9). Moreover, as already argued in Sec. III A 2, a pure bulk termination evolving upon heating from the cold DL structure seems physically improbable.

The metal-rich surfaces are significantly worse than the models discussed so far, the only exception being model (f), a pure Ti surface layer. The other three terminations result in much higher $\chi_{r}^{2}$ values and $R$ factors, in particular for the fits of the top 1 or 2 ALs, where improvements in the fit, assuming the model to be correct, should have the most influence. It can be perhaps argued that the reason that the Ti model (f) fits the experimental data a little better is because the $\mathrm{TiO}_{2}$-DL structure is also Ti-rich at the surface. Further investigations compared the two structures and gave consistently better results for the $\mathrm{TiO}_{2}$-DL model. Both models looked physically reasonable and had sensible DW factors, but the $\chi_{r}^{2}$ was always about $15 \%$ better for the $\mathrm{TiO}_{2}-\mathrm{DL}$ model, and any attempt to reach the same $\chi_{r}^{2}$ with model (f) failed. Another argument supporting the $\mathrm{TiO}_{2}$-DL model is that the cold structure did not exhibit any sign of a metal-rich surface. To obtain a metal-rich surface would therefore require comprehensive changes of the surface configuration upon annealing in a background of oxygen, which appears to be physically implausible.

\section{PARADIGM results}

The application of the PARADIGM algorithm on the hot data set recovers electron densities (ED) in an additional layer on top of the STO bulk unit cell, as shown in Fig. 10. The EDs are located at exactly the same positions as one would expect it for the $\mathrm{TiO}_{2}$-DL model, although with higher 
TABLE III. Refined positions and DW factors (expressed as root mean squared displacements in brackets) for the $(2 \times 1)$ reconstruction under cold conditions in units of bulk STO.

\begin{tabular}{|c|c|c|c|c|c|c|}
\hline $\mathrm{AL}$ & No. & Atom & $\begin{array}{l}\text { Nominal } \\
\text { position }\end{array}$ & $x / a_{0}$ & $y / a_{0}$ & $z / a_{0}$ \\
\hline \multirow[t]{6}{*}{1} & 1 & $\mathrm{Ti}$ & $\left(\begin{array}{lll}\frac{1}{2} & 0 & 3\end{array}\right)$ & $0.481(59)$ & $0.000(59)$ & $3.194(16)$ \\
\hline & 2 & $\mathrm{O}$ & $\left(\begin{array}{lll}0 & 0 & 3\end{array}\right)$ & $-0.005(20)$ & $0.000(20)$ & $3.119(132)$ \\
\hline & 3 & $\mathrm{O}$ & $\left(\begin{array}{lll}1 & 0 & 3\end{array}\right)$ & $1.032(20)$ & $0.000(20)$ & $2.975(20)$ \\
\hline & 4 & $\mathrm{Ti}$ & $\left(1 \frac{1}{2} 3\right)$ & $0.972(204)$ & $0.500(204)$ & $3.345(19)$ \\
\hline & 5 & $\mathrm{O}$ & $\left(\begin{array}{lll}\frac{1}{2} & \frac{1}{2} & 3\end{array}\right)$ & $0.584(57)$ & $0.500(57)$ & $3.260(40)$ \\
\hline & 6 & $\mathrm{O}$ & $\left(\begin{array}{lll}2 & \frac{1}{2} & 3\end{array}\right)$ & $1.500(44)$ & $0.500(44)$ & $3.266(20)$ \\
\hline \multirow[t]{6}{*}{2} & 7 & $\mathrm{Ti}$ & $\left(\begin{array}{lll}\frac{1}{2} & \frac{1}{2} & \frac{5}{2}\end{array}\right)$ & $0.536(56)$ & $0.500(56)$ & $2.564(14)$ \\
\hline & 8 & $\mathrm{O}$ & $\left(\begin{array}{lll}\frac{1}{2} & 0 & \frac{5}{2}\end{array}\right)$ & $0.575(20)$ & $0.000(20)$ & $2.637(20)$ \\
\hline & 9 & $\mathrm{O}$ & $\left(0 \frac{1}{2} \frac{5}{2}\right)$ & $0.017(20)$ & $0.500(20)$ & $2.627(20)$ \\
\hline & 10 & $\mathrm{Ti}$ & $\left(\begin{array}{lll}\frac{3}{2} & \frac{1}{2} & \frac{5}{2}\end{array}\right)$ & $1.590(67)$ & $0.500(67)$ & $2.647(70)$ \\
\hline & 11 & $\mathrm{O}$ & $\left(\begin{array}{lll}\frac{3}{2} & 0 & \frac{5}{2}\end{array}\right)$ & $1.485(20)$ & $0.000(20)$ & $2.570(20)$ \\
\hline & 12 & $\mathrm{O}$ & $\left(1 \frac{1}{2} \frac{5}{2}\right)$ & $0.964(109)$ & $0.500(109)$ & $2.579(20)$ \\
\hline \multirow[t]{4}{*}{3} & 13 & $\mathrm{Sr}$ & $\left(\begin{array}{lll}0 & 0 & 2\end{array}\right)$ & $0.016(34)$ & $0.000(34)$ & $2.020(58)$ \\
\hline & 14 & $\mathrm{O}$ & $\left(\begin{array}{lll}\frac{1}{2} & \frac{1}{2} & 2\end{array}\right)$ & $0.600(20)$ & $0.500(20)$ & $2.057(20)$ \\
\hline & 15 & $\mathrm{Sr}$ & $\left(\begin{array}{lll}1 & 0 & 2\end{array}\right)$ & $1.026(39)$ & $0.000(39)$ & $2.018(51)$ \\
\hline & 16 & $\mathrm{O}$ & $\left(\frac{3}{2} \frac{1}{2} 2\right)$ & $1.512(204)$ & $0.500(204)$ & $2.031(204)$ \\
\hline \multirow[t]{6}{*}{4} & 17 & $\mathrm{Ti}$ & $\left(\begin{array}{lll}\frac{1}{2} & \frac{1}{2} & \frac{3}{2}\end{array}\right)$ & $0.511(30)$ & $0.500(30)$ & $1.560(23)$ \\
\hline & 18 & $\mathrm{O}$ & $\left(\begin{array}{lll}\frac{1}{2} & 0 & \frac{3}{2}\end{array}\right)$ & $0.521(20)$ & $0.000(20)$ & $1.515(20)$ \\
\hline & 19 & $\mathrm{O}$ & $\left(0 \frac{1}{2} \frac{3}{2}\right)$ & $0.025(20)$ & $0.500(20)$ & 1.491(39) \\
\hline & 20 & $\mathrm{Ti}$ & $\left(\frac{3}{2} \quad \frac{1}{2} \frac{3}{2}\right)$ & $1.516(28)$ & $0.500(28)$ & $1.535(14)$ \\
\hline & 21 & $\mathrm{O}$ & $\left(\begin{array}{lll}\frac{3}{2} & 0 & \frac{3}{2}\end{array}\right)$ & $1.484(20)$ & $0.000(20)$ & $1.425(20)$ \\
\hline & 22 & $\mathrm{O}$ & $\left(1 \frac{1}{2} \frac{3}{2}\right)$ & $1.009(29)$ & $0.500(29)$ & $1.490(86)$ \\
\hline \multirow[t]{4}{*}{5} & 23 & $\mathrm{Sr}$ & $\left(\begin{array}{lll}0 & 0 & 1\end{array}\right)$ & $0.015(25)$ & $0.000(25)$ & $1.003(45)$ \\
\hline & 24 & $\mathrm{O}$ & $\left(\begin{array}{lll}\frac{1}{2} & \frac{1}{2} & 1\end{array}\right)$ & $0.499(88)$ & $0.500(88)$ & $1.008(140)$ \\
\hline & 25 & $\mathrm{Sr}$ & $\left(\begin{array}{lll}1 & 0 & 1\end{array}\right)$ & $1.010(25)$ & $0.000(25)$ & $1.007(37)$ \\
\hline & 26 & $\mathrm{O}$ & $\left(\begin{array}{lll}\frac{3}{2} & \frac{1}{2} & 1\end{array}\right)$ & $1.606(23)$ & $0.500(23)$ & $1.061(20)$ \\
\hline \multirow[t]{6}{*}{6} & 27 & $\mathrm{Ti}$ & $\left(\frac{1}{2} \frac{1}{2} \frac{1}{2}\right)$ & $0.489(27)$ & $0.500(27)$ & $0.506(27)$ \\
\hline & 28 & $\mathrm{O}$ & $\left(\begin{array}{lll}\frac{1}{2} & 0 & \frac{1}{2}\end{array}\right)$ & $0.547(50)$ & $0.000(50)$ & $0.499(50)$ \\
\hline & 29 & $\mathrm{O}$ & $\left(0 \frac{1}{2} \frac{1}{2}\right)$ & $-0.007(50)$ & $0.500(50)$ & $0.498(50)$ \\
\hline & 30 & $\mathrm{Ti}$ & $\left(\begin{array}{lll}\frac{3}{2} & \frac{1}{2} & \frac{1}{2}\end{array}\right)$ & $1.498(27)$ & $0.500(27)$ & $0.504(27)$ \\
\hline & 31 & $\mathrm{O}$ & $\left(\begin{array}{lll}\frac{3}{2} & 0 & \frac{1}{2}\end{array}\right)$ & $1.518(50)$ & $0.000(50)$ & $0.529(50)$ \\
\hline & 32 & $\mathrm{O}$ & $\left(1 \quad \frac{1}{2} \frac{1}{2}\right)$ & $1.020(50)$ & $0.500(50)$ & $0.525(50)$ \\
\hline
\end{tabular}

intensities on $\mathrm{O}$ than on the Ti positions. The agreement in the heights of recovered ED by PARADIGM (see Fig. 10) and the FIT results (see Table V) is striking. Therefore, these results strongly support the double layer model.

The data in Fig. 10 represents the averaged ED for two coherently added domains. ${ }^{55}$ The ratio of the recovered ED maxima between (a) and (b) is approximately 3:2. A possible explanation could involve the presence of $\mathrm{Sr}$ impurities on the surface, as it is indicated by our LEIS data (see Fig. 2). However, our attempts to model such impurities with FIT resulted in less than $1.5 \% \mathrm{Sr}$ remaining at the surface, clearly insufficient to account for this inversion of ED as seen by PARADIGM. The origin of the observed EDs therefore remains unclear.
PARADIGM may be applied to SXRD data from either coherently or incoherently scattering symmetrically related domains ${ }^{56-58}$ There is a probability that the present data arise from a mixture of coherently and incoherently scattering domains. The intensities of diffraction rods with $(h+k)$ even would be expected to be the same whether the domains scatter coherently or incoherently, and are therefore the most appropriate to be used as input to the algorithm in this case. On the other hand, the contributions to the intensities of these rods of even $(h+k)$ from the two domains of $p 2 \mathrm{~mm}$ symmetry rotated relative to each other by $90^{\circ}$ is the same. The Fourier transform of surface structure factors of even $(h+k)$ only will necessarily have $p 4 m m$ symmetry and may be identified with the average electron density of the two domains that is seen in Fig. 10.

The only contradiction between the surface electron density maps from PARADIGM seen in Fig. 10 and the best-fit model suggested by FIT is that the latter suggests the heavier atom $(\mathrm{Ti})$ to be associated with the smaller electron density features in the figure and that the lighter atom $(\mathrm{O})$ with the higher density. It is entirely possible that this apparent reversal of electron densities is due to the fact that an ideal substrate is assumed in the PARADIGM calculations, whereas FIT suggests significant reconstructions below the outermost double layer. Nevertheless, the model-independent nature of the PARADIGM result gives greater confidence to the conclusions of the trial-and-error fitting to the data of specific structural models.

\section{Final structure}

The SXRD data of STO under hot conditions is shown in Fig. 11. The best final surface structure has a crystallographic $R$ factor of $11.2 \%$ and no unphysical parameters. The structure is shown from the side in Fig. 7(d) ${ }^{54}$ It is very similar to the DL $(1 \times 1)$ structure for the cold data, but with more puckering of the $\mathrm{TiO}_{2} \mathrm{AL}$, as the topmost Ti atom moves further out of the surface.

In Table $\mathrm{V}$, the atomic coordinates are presented in the same manner as for the cold data. The uncertainty in the positions due to thermal effects is higher compared to the cold data, as one would expect. The displacements in the $z$ direction from high-symmetry positions in the starting models are further discussed in Sec. III F 1.

\section{DFT results}

The DFT results are summarized in Table VI. Seven models were tested, including the most promising DL structures. A comparison between our own calculation and recent work by other groups is also included. ${ }^{17,52,59}$

We focus first on the surface energies that were calculated using the PBE functional. The three lowest surface energies included the two reconstructions we found experimentally. As already mentioned, the DL $(2 \times 2)$ surface can be thought 
TABLE IV. Refined positions and DW factors (expressed as root mean squared displacements in brackets) for the $(2 \times 2)$ reconstruction under cold conditions in units of bulk STO.

\begin{tabular}{|c|c|c|c|c|c|c|c|c|c|c|c|c|c|}
\hline $\mathrm{AL}$ & No. & Atom & $\begin{array}{l}\text { Nominal } \\
\text { position }\end{array}$ & $x / a_{0}$ & $y / a_{0}$ & $z / a_{0}$ & $\mathrm{AL}$ & No. & Atom & $\begin{array}{l}\text { Nominal } \\
\text { position }\end{array}$ & $x / a_{0}$ & $y / a_{0}$ & $z / a_{0}$ \\
\hline \multirow[t]{12}{*}{1} & 1 & $\mathrm{Ti}$ & $\left(\begin{array}{lll}\frac{1}{2} & 0 & 3\end{array}\right)$ & $0.413(160)$ & $-0.055(160)$ & $2.920(98)$ & 4 & 33 & $\mathrm{Ti}$ & $\left(\begin{array}{lll}\frac{1}{2} & \frac{1}{2} & \frac{3}{2}\end{array}\right)$ & $0.525(59)$ & $0.500(59)$ & $1.478(152)$ \\
\hline & 2 & $\mathrm{O}$ & $\left(\begin{array}{lll}0 & 0 & 3\end{array}\right)$ & $0.062(20)$ & $0.112(20)$ & $3.015(93)$ & & 34 & $\mathrm{O}$ & $\left(\begin{array}{lll}\frac{1}{2} & 0 & \frac{3}{2}\end{array}\right)$ & $0.472(59)$ & $-0.013(59)$ & $1.487(204)$ \\
\hline & 3 & $\mathrm{O}$ & $\left(\begin{array}{lll}1 & 0 & 3\end{array}\right)$ & $1.378(67)$ & $-0.037(67)$ & $2.798(20)$ & & 35 & $\mathrm{O}$ & $\left(0 \frac{1}{2} \frac{3}{2}\right)$ & $-0.061(40)$ & $0.500(40)$ & $1.398(33)$ \\
\hline & 4 & $\mathrm{Ti}$ & $\left(\begin{array}{lll}0 & \frac{1}{2} & 3\end{array}\right)$ & $-0.085(73)$ & $0.500(73)$ & $3.013(14)$ & & 36 & $\mathrm{Ti}$ & $\left(\frac{3}{2} \quad \frac{1}{2} \frac{3}{2}\right)$ & $1.484(65)$ & $0.500(65)$ & $1.595(14)$ \\
\hline & 5 & $\mathrm{O}$ & $\left(\begin{array}{lll}2 & \frac{1}{2} & 3\end{array}\right)$ & $1.570(20)$ & $0.500(20)$ & $3.001(20)$ & & 37 & $\mathrm{O}$ & $\left(\begin{array}{lll}\frac{3}{2} & 0 & \frac{3}{2}\end{array}\right)$ & $1.415(67)$ & $-0.005(67)$ & $1.482(20)$ \\
\hline & 6 & $\mathrm{O}$ & $\left(\begin{array}{lll}\frac{1}{2} & \frac{1}{2} & 3\end{array}\right)$ & $0.534(29)$ & $0.500(29)$ & $2.885(20)$ & & 38 & $\mathrm{O}$ & $\left(1 \frac{1}{2} \frac{3}{2}\right)$ & $0.921(51)$ & $0.500(51)$ & $1.466(20)$ \\
\hline & 7 & $\mathrm{Ti}$ & $\left(\begin{array}{lll}\frac{1}{2} & 1 & 3\end{array}\right)$ & $0.413(160)$ & $1.055(160)$ & $2.920(98)$ & & 39 & $\mathrm{Ti}$ & $\left(\frac{1}{2} \frac{3}{2} \frac{3}{2}\right)$ & $0.571(64)$ & $1.500(64)$ & $1.349(111)$ \\
\hline & 8 & $\mathrm{O}$ & $\left(\begin{array}{lll}0 & 1 & 3\end{array}\right)$ & $0.062(20)$ & $0.888(20)$ & $3.015(93)$ & & 40 & $\mathrm{O}$ & $\left(\begin{array}{lll}\frac{1}{2} & 1 & \frac{3}{2}\end{array}\right)$ & $0.472(59)$ & $1.013(59)$ & $1.487(204)$ \\
\hline & 9 & $\mathrm{O}$ & $\left(\begin{array}{lll}1 & 1 & 3\end{array}\right)$ & $1.378(67)$ & $1.037(67)$ & $2.798(20)$ & & 41 & $\mathrm{O}$ & $\left(0 \frac{3}{2} \frac{3}{2}\right)$ & $-0.038(20)$ & $1.500(20)$ & $1.552(20)$ \\
\hline & 10 & $\mathrm{Ti}$ & $\left(1 \frac{3}{2} 3\right)$ & $0.988(58)$ & $1.500(58)$ & $3.005(62)$ & & 42 & $\mathrm{Ti}$ & $\left(\frac{3}{2} \frac{3}{2} \frac{3}{2}\right)$ & $1.614(59)$ & $1.500(59)$ & $1.440(83)$ \\
\hline & 11 & $\mathrm{O}$ & $\left(\frac{1}{2} \frac{3}{2} 3\right)$ & $0.527(20)$ & $1.500(20)$ & $2.746(20)$ & & 43 & $\mathrm{O}$ & $\left(\begin{array}{lll}\frac{3}{2} & 1 & \frac{3}{2}\end{array}\right)$ & $1.415(67)$ & $1.005(67)$ & $1.482(20)$ \\
\hline & 12 & $\mathrm{O}$ & $\left(\frac{3}{2} \frac{3}{2} 3\right)$ & $1.431(34)$ & $1.500(34)$ & $3.226(20)$ & & 44 & $\mathrm{O}$ & $\left(1 \quad \frac{3}{2} \frac{3}{2}\right)$ & $1.040(20)$ & $1.500(20)$ & $1.598(204)$ \\
\hline \multirow[t]{12}{*}{2} & 13 & $\mathrm{Ti}$ & $\left(\begin{array}{lll}\frac{1}{2} & \frac{1}{2} & \frac{5}{2}\end{array}\right)$ & $0.645(14)$ & $0.500(14)$ & $2.238(129)$ & 5 & 45 & $\mathrm{Sr}$ & $\left(\begin{array}{lll}0 & 0 & 1\end{array}\right)$ & $-0.032(78)$ & $0.003(78)$ & $1.010(76)$ \\
\hline & 14 & $\mathrm{O}$ & $\left(\begin{array}{lll}\frac{1}{2} & 0 & \frac{5}{2}\end{array}\right)$ & $0.413(30)$ & $-0.071(30)$ & $2.436(20)$ & & 46 & $\mathrm{O}$ & $\left(\begin{array}{lll}\frac{1}{2} & \frac{1}{2} & 1\end{array}\right)$ & $0.454(59)$ & $0.500(59)$ & $0.986(88)$ \\
\hline & 15 & $\mathrm{O}$ & $\left(0 \frac{1}{2} \frac{5}{2}\right)$ & $-0.045(38)$ & $0.500(38)$ & $2.384(108)$ & & 47 & $\mathrm{Sr}$ & $\left(\begin{array}{lll}1 & 0 & 1\end{array}\right)$ & $0.994(77)$ & $-0.001(77)$ & $1.012(96)$ \\
\hline & 16 & $\mathrm{Ti}$ & 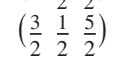 & $1.584(48)$ & $0.500(48)$ & $2.642(14)$ & & 48 & $\mathrm{O}$ & $\left(\begin{array}{lll}\frac{3}{2} & \frac{1}{2} & 1\end{array}\right)$ & 1.488(97) & $0.500(97)$ & $1.014(60)$ \\
\hline & 17 & $\mathrm{O}$ & $\left(\begin{array}{lll}\frac{3}{2} & 0 & \frac{5}{2}\end{array}\right)$ & $1.473(61)$ & $0.016(61)$ & $2.499(70)$ & & 49 & $\mathrm{Sr}$ & $\left(\begin{array}{lll}0 & 1 & 1\end{array}\right)$ & $-0.032(78)$ & $0.997(78)$ & $1.010(76)$ \\
\hline & 18 & $\mathrm{O}$ & $\left(\begin{array}{lll}1 & \frac{1}{2} & \frac{5}{2}\end{array}\right)$ & $0.925(20)$ & $0.500(20)$ & $2.437(204)$ & & 50 & $\mathrm{O}$ & $\left(\begin{array}{lll}1 & \frac{3}{2} & 1\end{array}\right)$ & $0.422(20)$ & $1.500(20)$ & $1.020(100)$ \\
\hline & 19 & $\mathrm{Ti}$ & 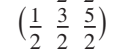 & $0.427(44)$ & $1.500(44)$ & $2.343(14)$ & & 51 & $\mathrm{Sr}$ & $\left(\begin{array}{lll}1 & 1 & 1\end{array}\right)$ & $0.994(77)$ & $1.001(77)$ & $1.012(96)$ \\
\hline & 20 & $\mathrm{O}$ & $\left(\begin{array}{lll}\frac{1}{2} & 1 & \frac{5}{2}\end{array}\right)$ & $0.413(30)$ & $1.071(30)$ & $2.436(20)$ & & 52 & $\mathrm{O}$ & $\left(\frac{3}{2} \frac{3}{2} 1\right)$ & $1.539(45)$ & $1.500(45)$ & $1.064(20)$ \\
\hline & 21 & $\mathrm{O}$ & $\left(0 \frac{3}{2} \frac{5}{2}\right)$ & $-0.025(20)$ & $1.500(20)$ & $2.607(90)$ & 6 & 53 & $\mathrm{Ti}$ & $\left(\begin{array}{lll}\frac{1}{2} & \frac{1}{2} & \frac{1}{2}\end{array}\right)$ & $0.504(41)$ & $0.500(41)$ & $0.541(41)$ \\
\hline & 22 & $\mathrm{Ti}$ & $\left(\frac{3}{2} \frac{3}{2} \frac{5}{2}\right)$ & $1.479(100)$ & $1.500(100)$ & $2.631(158)$ & & 54 & $\mathrm{O}$ & $\left(\begin{array}{lll}\frac{1}{2} & 0 & \frac{1}{2}\end{array}\right)$ & $0.444(31)$ & $-0.004(31)$ & $0.538(31)$ \\
\hline & 23 & $\mathrm{O}$ & $\left(\begin{array}{lll}\frac{3}{2} & 1 & \frac{5}{2}\end{array}\right)$ & $1.473(61)$ & $0.984(61)$ & $2.499(70)$ & & 55 & $\mathrm{O}$ & $\left(\begin{array}{lll}0 & \frac{1}{2} & \frac{1}{2}\end{array}\right)$ & $0.012(31)$ & $0.500(31)$ & $0.528(31)$ \\
\hline & 24 & $\mathrm{O}$ & 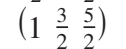 & $0.953(79)$ & $1.500(79)$ & $2.518(20)$ & & 56 & $\mathrm{Ti}$ & $\left(\begin{array}{lll}3 & \frac{1}{2} & \frac{1}{2}\end{array}\right)$ & $1.516(41)$ & $0.500(41)$ & $0.535(41)$ \\
\hline \multirow[t]{8}{*}{3} & 25 & $\mathrm{Sr}$ & $\left(\begin{array}{lll}0 & 0 & 2\end{array}\right)$ & $-0.012(81)$ & $-0.006(81)$ & $2.018(149)$ & & 57 & $\mathrm{O}$ & $\left(\begin{array}{lll}\frac{3}{2} & 0 & \frac{1}{2}\end{array}\right)$ & $1.475(31)$ & $-0.004(31)$ & $0.528(31)$ \\
\hline & 26 & $\mathrm{O}$ & $\left(\begin{array}{lll}\frac{1}{2} & \frac{1}{2} & 2\end{array}\right)$ & $0.424(37)$ & $0.500(37)$ & $1.862(20)$ & & 58 & $\mathrm{O}$ & $\left(1 \quad \frac{1}{2} \frac{1}{2}\right)$ & $0.994(31)$ & $0.500(31)$ & $0.532(31)$ \\
\hline & 27 & $\mathrm{Sr}$ & $\left(\begin{array}{lll}1 & 0 & 2\end{array}\right)$ & $0.935(98)$ & $0.006(98)$ & $2.039(100)$ & & 59 & $\mathrm{Ti}$ & $\left(\begin{array}{lll}1 & \frac{1}{2} & \frac{1}{2}\end{array}\right)$ & $0.516(41)$ & $1.500(41)$ & $0.514(41)$ \\
\hline & 28 & $\mathrm{O}$ & $\left(\frac{3}{2} \frac{1}{2} 2\right)$ & $1.481(79)$ & $0.500(79)$ & $1.964(20)$ & & 60 & $\mathrm{O}$ & $\left(\begin{array}{lll}\frac{1}{2} & 1 & \frac{1}{2}\end{array}\right)$ & $0.444(31)$ & $1.004(31)$ & $0.538(31)$ \\
\hline & 29 & $\mathrm{Sr}$ & $\left(\begin{array}{lll}0 & 1 & 2\end{array}\right)$ & $-0.012(81)$ & $1.006(81)$ & $2.018(149)$ & & 61 & $\mathrm{O}$ & $\left(0 \frac{3}{2} \frac{1}{2}\right)$ & $0.004(31)$ & $1.500(31)$ & $0.555(31)$ \\
\hline & 30 & $\mathrm{O}$ & $\left(\frac{1}{2} \frac{3}{2} 2\right)$ & $0.356(46)$ & $1.500(46)$ & $1.973(20)$ & & 62 & $\mathrm{Ti}$ & $\left(\frac{3}{2} \frac{3}{2} \frac{1}{2}\right)$ & $1.530(41)$ & $1.500(41)$ & $0.509(41)$ \\
\hline & 31 & $\mathrm{Sr}$ & $\left(\begin{array}{lll}1 & 1 & 2\end{array}\right)$ & $0.935(98)$ & $0.994(98)$ & $2.039(100)$ & & 63 & $\mathrm{O}$ & $\left(\begin{array}{lll}\frac{3}{2} & 1 & \frac{1}{2}\end{array}\right)$ & $1.475(31)$ & $1.004(31)$ & $0.528(31)$ \\
\hline & 32 & $\mathrm{O}$ & $\left(\frac{3}{2} \frac{3}{2} 2\right)$ & $1.503(204)$ & $1.500(204)$ & $2.064(22)$ & & 64 & $\mathrm{O}$ & $\left(\begin{array}{lll}1 & \frac{3}{2} & \frac{1}{2}\end{array}\right)$ & $0.993(31)$ & $1.500(31)$ & $0.566(31)$ \\
\hline
\end{tabular}

as consisting of alternately flipped DL $(2 \times 1)$ surface cells, and hence their similar chemistry explains their comparable surface energies.

The other low-energy configuration is a bulk $(1 \times 1)$, whereas the experimentally found DL $(1 \times 1)$ structure is significantly higher in energy. As we will show later, this may be explained by a (possibly dynamic) order-disorder transition. Our attempts to model the terminations against a wet medium instead of vacuum led to energies that were about $15 \%$ lower (see column " $e$-wet" in Table VI).

The surface energies of the two most promising models for the $(2 \times 2)$ DL structures were calculated [see Figs. 5(a) and 5(b)]. Although the top AL of both models differ only by a lateral shift of $(1 / 21 / 2)$ bulk STO cell, the surface energy of model (a) is lower (see LDA-PWC column, Table VI). This is because a $(1 / 21 / 2)$ shift means that the bonding to the next AL below is fundamentally different.

Heifets et al. suggested recently that the surface of STO in the thermodynamical equilibrium should be terminated with SrO. ${ }^{59}$ Experimentally, such $\mathrm{SrO}$ terminations are typically obtained if the sample is not chemically treated (in a buffered $\mathrm{HF}$ solution), but annealed at temperatures above $1000{ }^{\circ} \mathrm{C}$ for $24 \mathrm{~h}$ to several days in oxygen. ${ }^{31,60,61}$ In our DFT work, we did not calculate surface energies for SrO terminations. Comparing the $\mathrm{TiO}_{2}$-terminated surfaces in Table VI, we see that the energies are in very good agreement with our values calculated using the LDA-PWC functional. Thus, if thermodynamical equilibrium is not reached, the presence of kineti- 


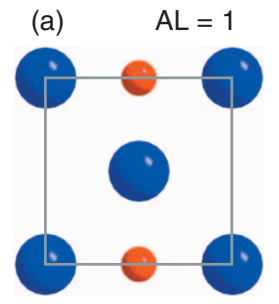

$\mathrm{p} 2 \mathrm{~mm}$

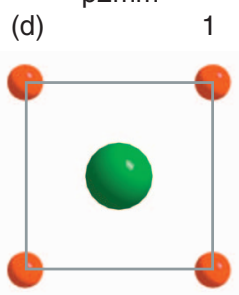

$\mathrm{p} 4 \mathrm{~mm}$ (b)

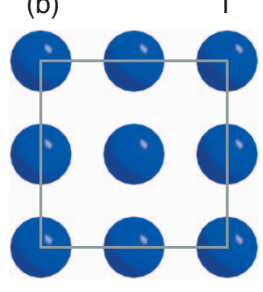

$\mathrm{p} 4 \mathrm{~mm}$

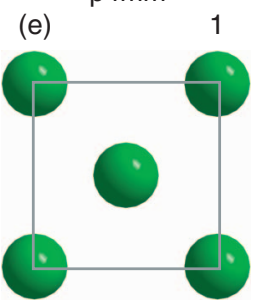

$\mathrm{p} 4 \mathrm{~mm}$

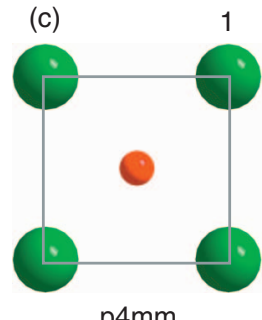

$\mathrm{p} 4 \mathrm{~mm}$

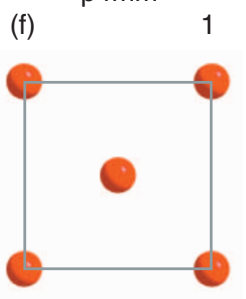

$\mathrm{p} 4 \mathrm{~mm}$
FIG. 8. (Color) Hot $(1 \times 1)$ starting models using the same representation as in Fig. 3.

cally favored $\mathrm{TiO}_{2}$-terminated surfaces remains an interesting suggestion and is compatible with the experimental observations.

Our DFT results indicate that the $(2 \times 1)$ DL reconstruction should be equally favored as bulk-terminated STO (in contrast to the results presented by Johnston et al. ${ }^{17}$ ), and that the $(2 \times 2)$ DL reconstruction should be most favored.

If we compare the energies obtained using the GGE-PBE functional with the results of Warschkow et al., ${ }^{52}$ we see a consistent trend for the difference between the $(1 \times 1)-\mathrm{DL}$ surface energy and those of the $(2 \times 1)$-DL and $(2 \times 2)$-DL reconstructions, which confirms the very low surface energies for the $(2 \times 1)$ and $(2 \times 2)$ DL reconstructions. It is

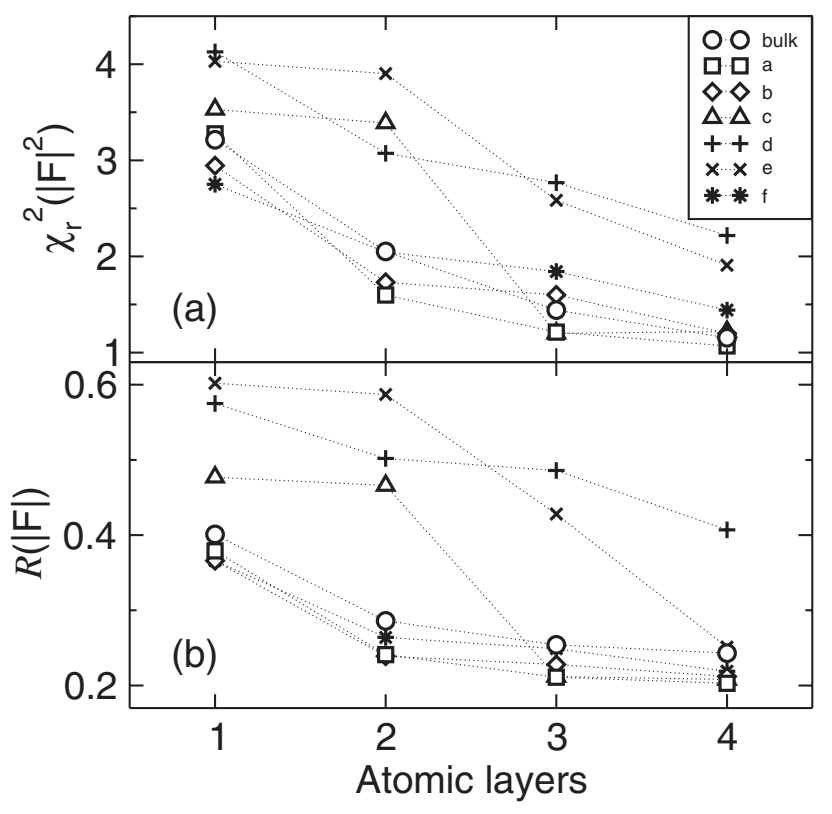

FIG. 9. The evolution of $\chi_{r}^{2}$ (a) and $R$ factor (b) as a function of the fit ALs for hot STO under identical conditions and started from the same initial positions. The dotted lines are guides to the eyes.
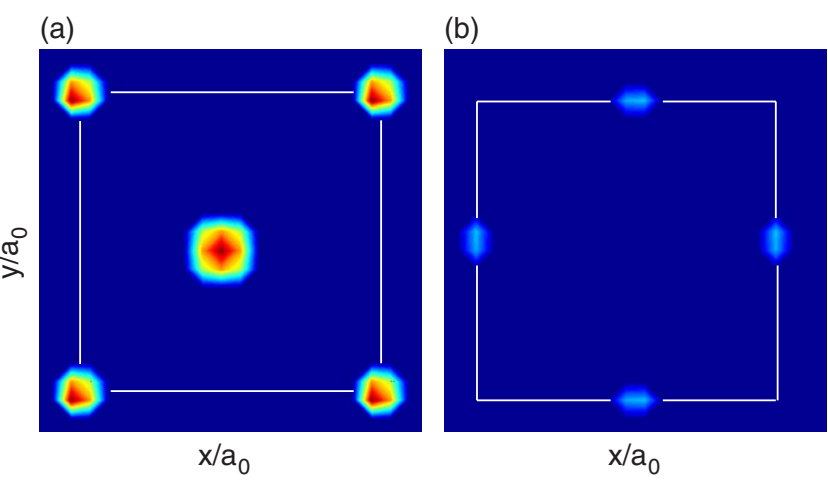

FIG. 10. (Color online) Cuts through the average electron density recovered by PARADIGM for coherently added domains at two different heights: $\mathrm{O}$ positions at $z / a_{0}=2.97$ (a) and Ti positions at $z / a_{0}=3.28$ (b). Representation is in bulk units of STO and analogously to Table V, for direct comparison. The white line indicates the STO bulk unit cell.

noted that the $(2 \times 2)$ reconstruction observed in this work is among the three surfaces with the lowest absolute energy. The $c(4 \times 2)$ has been experimentally observed by annealing in (i) $\mathrm{O}_{2}$ at temperatures of $850-930{ }^{\circ} \mathrm{C}$, ${ }^{62}$ (ii) $\mathrm{H}_{2}$ at $950{ }^{\circ} \mathrm{C},{ }^{10}$ and (iii) in UHV after Ar ion sputtering at a temperature of $1200{ }^{\circ} \mathrm{C},{ }^{15}$ while the $(\sqrt{2} \times \sqrt{2}) R 45^{\circ}$ reconstruction has yet to be experimentally observed.

\section{Formation of reconstructions}

A possible explanation for the driving force of the formation of the reconstructions could invoke the role of $\mathrm{Ca}$ im-

TABLE V. Refined positions and DW factors (expressed as root mean squared displacements in brackets) for the $(1 \times 1)$ reconstruction under hot conditions in units of bulk STO.

\begin{tabular}{|c|c|c|c|c|c|c|}
\hline $\mathrm{AL}$ & No. & Atom & $\begin{array}{l}\text { Nominal } \\
\text { position }\end{array}$ & $x / a_{0}$ & $y / a_{0}$ & $z / a_{0}$ \\
\hline \multirow[t]{6}{*}{1} & 1 & $\mathrm{Ti}$ & $\left(\begin{array}{lll}\frac{1}{2} & 0 & 3\end{array}\right)$ & $0.500(204)$ & $0.000(204)$ & $3.256(204)$ \\
\hline & 2 & $\mathrm{O}$ & $\left(\begin{array}{lll}0 & 0 & 3\end{array}\right)$ & $0.000(71)$ & $0.000(71)$ & $2.990(29)$ \\
\hline & 3 & $\mathrm{O}$ & $\left(\begin{array}{lll}\frac{1}{2} & \frac{1}{2} & 3\end{array}\right)$ & $0.500(204)$ & $0.500(204)$ & $3.102(204)$ \\
\hline & 4 & $\mathrm{Ti}$ & $\left(\begin{array}{lll}1 & \frac{1}{2} & \frac{5}{2}\end{array}\right)$ & $0.500(35)$ & $0.500(35)$ & $2.574(118)$ \\
\hline & 5 & $\mathrm{O}$ & $\left(\begin{array}{lll}\frac{1}{2} & 0 & \frac{5}{2}\end{array}\right)$ & $0.500(125)$ & $0.000(125)$ & $2.616(22)$ \\
\hline & 6 & $\mathrm{O}$ & $\left(\begin{array}{lll}0 & \frac{1}{2} & \frac{5}{2}\end{array}\right)$ & $0.000(20)$ & $0.500(20)$ & $2.485(62)$ \\
\hline \multirow[t]{2}{*}{3} & 7 & $\mathrm{Sr}$ & $\left(\begin{array}{lll}0 & 0 & 2\end{array}\right)$ & $0.000(45)$ & $0.000(45)$ & $2.009(44)$ \\
\hline & 8 & $\mathrm{O}$ & $\left(\begin{array}{lll}\frac{1}{2} & \frac{1}{2} & 2\end{array}\right)$ & $0.500(143)$ & $0.500(143)$ & $1.929(41)$ \\
\hline \multirow[t]{3}{*}{4} & 9 & $\mathrm{Ti}$ & $\left(\begin{array}{lll}\frac{1}{2} & \frac{1}{2} & \frac{3}{2}\end{array}\right)$ & $0.500(27)$ & $0.500(27)$ & $1.506(14)$ \\
\hline & 10 & $\mathrm{O}$ & $\left(\begin{array}{lll}\frac{1}{2} & 0 & \frac{3}{2}\end{array}\right)$ & $0.500(22)$ & $0.000(22)$ & $1.545(20)$ \\
\hline & 11 & $\mathrm{O}$ & $\left(\begin{array}{lll}0 & \frac{1}{2} & \frac{3}{2}\end{array}\right)$ & $0.000(20)$ & $0.500(20)$ & $1.410(20)$ \\
\hline \multirow[t]{2}{*}{5} & 12 & $\mathrm{Sr}$ & $\left(\begin{array}{lll}0 & 0 & 1\end{array}\right)$ & $0.000(36)$ & $0.000(36)$ & $1.004(41)$ \\
\hline & 13 & $\mathrm{O}$ & $\left(\begin{array}{lll}\frac{1}{2} & \frac{1}{2} & 1\end{array}\right)$ & $0.500(51)$ & $0.500(51)$ & $0.947(44)$ \\
\hline \multirow[t]{3}{*}{6} & 14 & $\mathrm{Ti}$ & $\left(\begin{array}{lll}\frac{1}{2} & \frac{1}{2} & \frac{1}{2}\end{array}\right)$ & $0.500(26)$ & $0.500(26)$ & $0.502(26)$ \\
\hline & 15 & $\mathrm{O}$ & $\left(\begin{array}{lll}\frac{1}{2} & 0 & \frac{1}{2}\end{array}\right)$ & $0.500(43)$ & $0.000(43)$ & $0.510(43)$ \\
\hline & 16 & $\mathrm{O}$ & $\left(\begin{array}{lll}0 & \frac{1}{2} & \frac{1}{2}\end{array}\right)$ & $0.000(43)$ & $0.500(43)$ & $0.474(43)$ \\
\hline
\end{tabular}




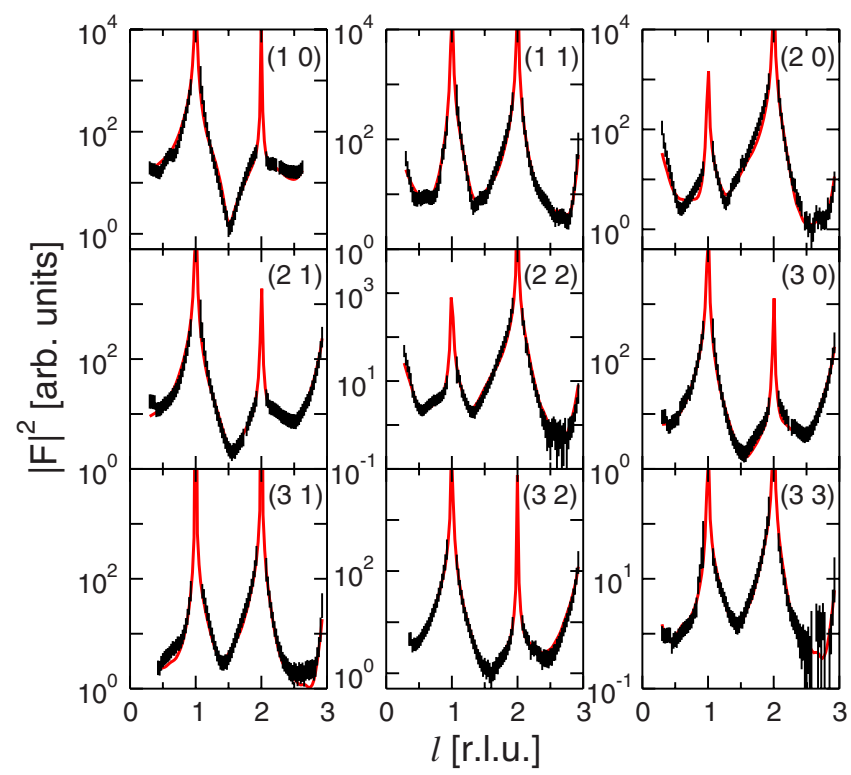

FIG. 11. (Color online) Set of the SXRD data (black data points) and calculated intensities using the $\mathrm{TiO}_{2}$-DL model (gray solid line, red online) for the hot structure.

purities. On the one hand, these have been demonstrated to be of great importance to reconstruct and modify surfaces for a variety of oxides, including SrO-terminated $\operatorname{STO}(001){ }^{6}$ $\mathrm{MgO}(001),{ }^{63,64} \mathrm{TiO}_{2}(110),{ }^{65}$ and $\mathrm{Fe}_{3} \mathrm{O}_{4}(001) .{ }^{66}$ On the other hand, trace amounts of $\mathrm{Ca}$ are a source of impurities in the production of single-crystalline $\mathrm{STO} .^{22}$

Of particular interest in this context is the work by Andersen and Møller, ${ }^{6}$ where they used Auger electron spectroscopy and low-energy electron-diffraction to observe the formation of an overlayer structure (with $\mathrm{Ca}$ incorporated in the surface) for a Ca content of $3 \%$, and a $(2 \times 2)$ reconstruction when the $\mathrm{Ca}$ content was below $1 \%$. This reconstruction later disappeared completely when the Ca was fully removed. It is noteworthy that they judged this $(2 \times 2)$ reconstruction to penetrate at most 3 to 4 MLs into the bulk.

The LEIS spectra shown in Fig. 2 suggest a similar behavior of $\mathrm{TiO}_{2}$-terminated STO: As-received samples contain a higher amount of $\mathrm{Ca}$ at the surface and show no reconstructions. The chemical and thermal treatment of the surface according to Refs. 23 and 24 may lead to a decreased Ca content in the surface that allows the formation of reconstructions, as observed by us. A further etch cycle, as suggested by Ohnishi et al. ${ }^{27}$ diminishes the Ca content further and may result in the complete absence of reconstructions again. This is, indeed, what we observed: Not all of the re-etched STO samples showed reconstructions. There seems to be a direct correlation between the presence of reconstructions and the $\mathrm{Ca}$ impurities, the amount of the latter determined by both the intrinsic Ca content of STO single crystals and the etching conditions.

The LEIS spectra show further an amount of Sr present in the outermost layer (see Fig. 2). Here one has to take into account that the $\mathrm{Sr}$ signal compared to Ti is enhanced by a factor of 1.7 due to the different mass of the nuclei that change their detection probabilities accordingly. ${ }^{67}$ Moreover, even a modest treatment of the surface with pure water or buffered HF removes residing $\mathrm{Sr}$ contaminations. ${ }^{27}$ We note that in our case the re-etch procedure did not remove all the residuals on the sample on which LEIS was performed. ${ }^{68}$ The green spectrum in Fig. 2 shows among Sr also a significant $\mathrm{F}$ peak, an impurity attesting to possibly incomplete purification: the $\mathrm{F}$ peak is likely due to the buffer salt $\left(\mathrm{NH}_{4} \mathrm{~F}\right)$. Although the samples we used for SXRD did not undergo an additional etching step to remove any residuals, it seems likely that the use of highly brilliant synchrotron radiation has the same effect. In any case, our attempts to model the surface with additional $\mathrm{Sr}$ at the surface failed.

TABLE VI. DFT surface energies in $\mathrm{eV} /(1 \times 1)$ unit cell (with respect to the SrO chemical potential) for selected models. The letters in brackets identify the models in the corresponding figures.

\begin{tabular}{|c|c|c|c|c|c|c|c|}
\hline \multirow{3}{*}{$\begin{array}{c}\text { Functional } \\
\text { STO bulk unit cells }\end{array}$} & \multicolumn{4}{|c|}{ Present work } & \multirow{3}{*}{$\frac{\text { Heifets }^{\mathrm{a}}}{\text { B3PW-PP }}$} & \multirow{3}{*}{ 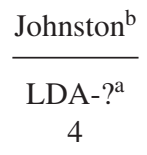 } & \multirow{3}{*}{$\begin{array}{c}\text { Warschkow } \\
\text { GGA-91 } \\
2\end{array}$} \\
\hline & LDA-PWC & GGA-PBE & $e$-wet & GGA-PBE & & & \\
\hline & 6 & 6 & 6 & 4 & & & \\
\hline$(1 \times 1)$ bulk, $(a)$ and $(b)$ & 1.02 & 0.78 & 0.67 & & 1.05 & 1.28 & \\
\hline$(1 \times 1) \mathrm{DL}(\mathrm{c})$ & 1.64 & 1.39 & 1.26 & & 1.75 & & $0^{\mathrm{e}}$ \\
\hline$(2 \times 1)$ DL (a) & 1.31 & 0.78 & 0.63 & 0.81 & 1.3 & 2.00 & -0.10 \\
\hline$(2 \times 2) \mathrm{DL}(\mathrm{a})$ & 0.79 & 0.38 & 0.33 & & & & -0.66 \\
\hline$(2 \times 2) \mathrm{DL}(\mathrm{b})$ & 1.22 & & & & & & -0.16 \\
\hline$c(4 \times 2)$ & & & & 0.49 & & & -0.64 \\
\hline$(\sqrt{2} \times \sqrt{2}) R 45^{\circ}$ & & & & 0.33 & & & -0.79 \\
\hline
\end{tabular}

${ }^{a}$ Reference 60 from Fig. 6(a) therein.

${ }^{\mathrm{b}}$ Reference 17 from Figs. 4 and 5(a) therein.

${ }^{\mathrm{c}}$ Reference 52.

${ }^{\mathrm{d}}$ Exact functional not specified.

eSubsequent values refer to this configuration as having zero energy. 


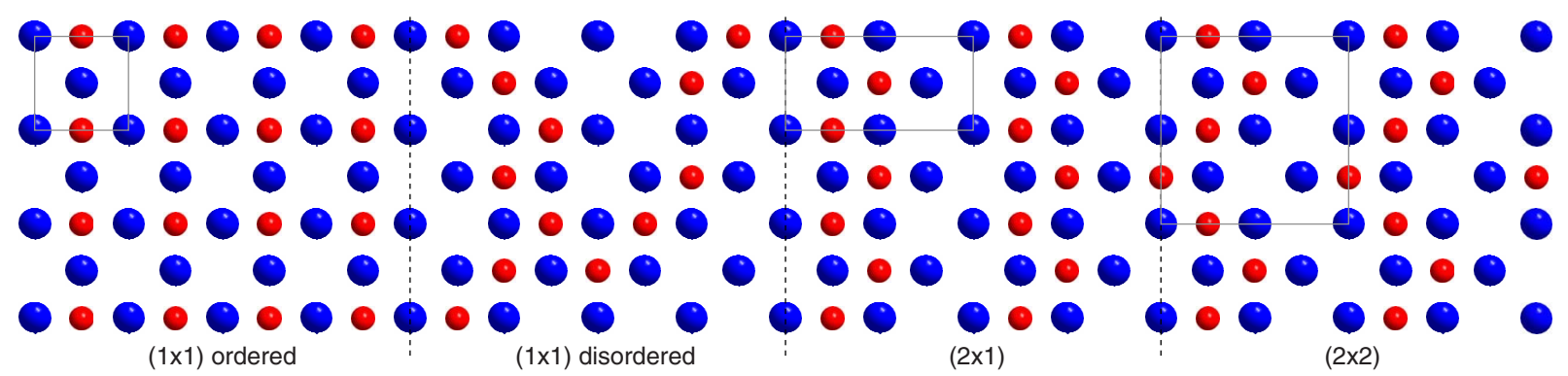

FIG. 12. (Color online) A schematic showing the similarity and simple transformation between the top layers of either the (ordered) $(1 \times 1),(2 \times 1)$, and $(2 \times 2)$ terminations. Transformation of the ordered $(1 \times 1)$-DL structure and either reconstruction only requires a diagonal movement of every second Ti atom, with the possibility of forming a disordered $(1 \times 1)$ relaxation ("grain boundaries") in between the domains. The gray boxes indicate the surface unit cells of the terminations. Color code: Ti small red, O large blue circles.

\section{E. Order-disorder transition}

Transformation between the DL $(1 \times 1)$ relaxation and either reconstruction only requires a diagonal hop of every second $\mathrm{Ti}$ atom across half a surface $(1 \times 1)$ unit cell, as schematically shown in Fig. 12. The surface energy of the $(1 \times 1)$ DL structure seems, however, anomalously high (see Table VI). The experimentally determined presence of a $(1 \times 1)$ structure could have two reasons: either this $(1 \times 1)$ domain is metastable with a significant activation barrier to lower-energy states, or in fact it consists of a disordered mixture of the characteristic zigzag motif of the $(2 \times 1)$ and $(2 \times 2)$, randomly flipped and mirrored. ${ }^{69}$ This (possibly dynamically) disordered $(1 \times 1)$ domain would have a surface energy approximately as low as the two reconstructions, and the average structure observed via SXRD could be described by the $\mathrm{TiO}_{2} \mathrm{DL}(1 \times 1)$. Such a model would not have to invoke coordinated and concerted Ti-hopping across the surface over large distances (of the order of some $100 \mathrm{~nm}$ ) to go from the reconstructions to the $(1 \times 1)$ relaxation. Disorder could also explain how such a $(1 \times 1)$ surface can dominate at elevated temperatures, where the surface thermal vibrational energy becomes comparable to the activation barrier between the $(2 \times 1)$ and $(2 \times 2)$, leading to a disordered mixture. Indeed, the difference in surface energy between two $(2 \times 1)$ cells and a single $(2 \times 2)$ cell is $\Delta E_{s}=1.6 \mathrm{eV}$ (see Table VI). On the other hand, the vibrational energy of the surface atoms of the same system is, to a first approximation, equal to $E_{v}=3 k T \times\left(4 n_{a}\right)$, where $k$ is Boltzmann's constant, $T$ is the temperature, $n_{a}$ is the number of atoms per top sublayer and unit cell (here, for $\mathrm{TiO}_{2}, n_{a}=3$ ), and the factor 4 accounts for the fact that 4 surface $(1 \times 1)$ cells are needed to describe this system. We therefore obtain $E_{v}=3.2 \mathrm{eV}$ under hot conditions, i.e., twice the difference in reconstruction surface energies. The size of the activation barrier between the two reconstructions will affect the time needed to reach this mixed equilibrium state. From the Arrhenius rate constant of the disappearance of the SSR signal of the order of $k_{s}=0.01 \mathrm{~s}^{-1}$, and assuming a preexponential factor $A \sim 10^{12}$, we obtain an activation energy of $E_{a} \approx 3 \mathrm{eV}$. For the room temperature sample, $E_{v}=0.9 \mathrm{eV}$ and the reaction rate constant is of the order of $10^{-40} \mathrm{~s}^{-1}$, i.e., the system is completely kinetically hindered. On a speculative note, the dis- ordered $(1 \times 1)$ domains suggested here may, in the cold sample, comprise regions, or "grain boundaries" of disorder between the ordered $(2 \times 1)$ and $(2 \times 1)$ reconstructions, frozen in as the sample cools at the end of the substrate preparation procedure.

\section{F. Electrostatic considerations}

\section{Atomic displacements}

In Fig. 13, we show the displacements of the atomic positions $\Delta z$ in the final model compared to the high-symmetry positions in the starting models. The displacements are most prominent in the $z$ direction (i.e., normal to the surface), and, for the $(1 \times 1)$ relaxations, movements only along this axis are allowed, due to the surface symmetry.

Our data show significant atomic movements for all refined layers down to a depth of three unit cells, the uppermost atoms on average displaced outwards to the vacuum. This implies a loss of centrosymmetry of the surface Tiatoms within the $\mathrm{O}$ octahedra and may lead to a permanent

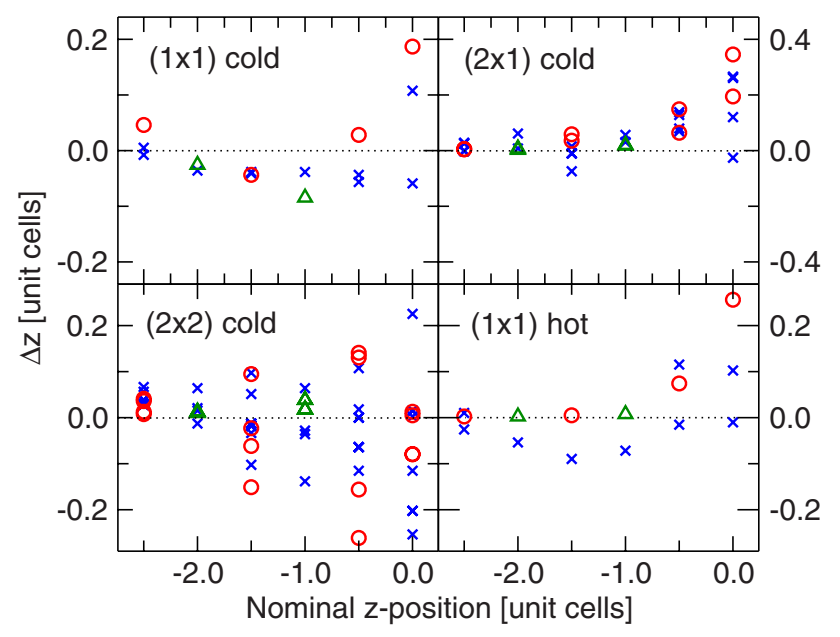

FIG. 13. (Color online) Displacements of $\mathrm{Sr}$ (green triangles), $\mathrm{Ti}$ (red circles), and $\mathrm{O}$ (blue crosses) in the $z$ direction from the highsymmetry positions in the starting models. The nominal surface is at $z=0$ and positive values of $\Delta z$ indicate displacements towards vacuum. 
surface dipole moment and surface ferroelectricity. $2,70,71$

The trends shown in our experimental data for the $(2 \times 1)$ DL structure are very similar to those theoretically predicted by Johnston et al. ${ }^{17}$ Therefore, theoretical and experimental arguments both support the puckering of the uppermost Ti sites in STO.

We note that the atomic movements in the $z$ direction for the $(2 \times 1)$ and the $(2 \times 2)$ reconstruction are different. This is a surprising result under consideration of the chemical similarity and the proposed formation mechanism of the two reconstructions. With respect to Fig. 7 and Tables III and IV, we see more pronounced movements of the atoms in the $x y$ plane of the $(2 \times 2)$ reconstruction. A possible explanation could involve symmetry arguments as follows: The pm symmetry applied to the $(2 \times 2)$ unit cell allows movements in the $y$ direction for certain atoms whereas this symmetry assigned to a $(2 \times 1)$ reconstruction prohibits such shifts completely. Therefore, the atoms of the $(2 \times 2)$ reconstruction are less constrained to movements out of the surface plane, but the additional freedom of $y$ movements may help obtaining a low surface energy. ${ }^{72}$

\section{Polar surfaces}

According to classical electrostatics, the stability of a crystal surface depends on the charge distribution in the unit cell in the direction perpendicular to the surface. Polar oxide surfaces can be classified into three different types. ${ }^{73}$ Type 1 surfaces have neither a net charge $\sigma$ nor a net dipole moment $\mu$ perpendicular to the surface. A typical example would be the $\mathrm{MgO}(001)$ surface that consists of layers containing equal amounts of $\mathrm{Mg}$ and $\mathrm{O}$ atoms. The type 2 surface displays a nonzero charge $\sigma \neq 0$, but has no net dipole moment within the unit cell in the normal direction, $\mu=0$, e.g., a $\mathrm{TiO}_{2}(110)$ surface. Because $\mu=0$, these two surface types are called nonpolar and are potentially stable. In a polar type 3 surface, $\sigma \neq 0$ and $\mu \neq 0$ in the repeat units of the structure. An example of a type 3 surface is $\mathrm{MgO}(111)$. Assuming ionic bonding, the total dipole moment of repeated units normal to the surface is directly proportional to the slab thickness, and therefore, the surface energy per unit area diverges even for thin films. This is the origin of the surface instability of type 3 surfaces.

An STO(001) perovskite crystal consists of repeated $\mathrm{TiO}_{2}$ and $\mathrm{SrO}$ layers, and one might assume it to be of type 1 , because the formal charges of $\mathrm{Ti}^{4+}, \mathrm{Sr}^{2+}$, and $\mathrm{O}^{2-}$ are compensated layer by layer and no net dipole moment is present. However, STO is not fully ionic. The partially covalent nature of the bonds (a property later referred to as "back transfer") in STO results in ALs having nonzero and opposite net charges $\pm \sigma .{ }^{51}$ Thus, $\mathrm{STO}(001)$ is a "weakly polar" type 3 surface. For polar systems with only a single distorted layer, this top $\mathrm{AL}$ is required to have a charge of $\sigma^{\prime}=\sigma / 2$ in order to avoid instability.

One can now calculate the net charge per unit area for a bulk-coordinated unit cell of STO using a covalency-induced back transfer of 0.422 electrons $\left(e^{-}\right)$per Ti and $0.045 e^{-}$per Sr site, respectively. ${ }^{74}$ Taking the coordination of the different atoms into account, this results in a net charge per unit cell area of $\sigma a_{0}^{2}=-0.484 e^{-}$for a $\mathrm{TiO}_{2} \mathrm{AL}$ that is compensated by $\sigma a_{0}^{2}=+0.484 e^{-}$of the SrO AL, i.e., STO is indeed a polar system. The terminating $\mathrm{TiO}_{2}$ layer of bulk STO has to meet the condition of $\sigma^{\prime}=\sigma / 2$ to be stable. Simple calculations show that for the reduced number of bonds of a topmost $\mathrm{TiO}_{2}$ layer $\sigma^{\prime} a_{0}^{2}=-0.242 e^{-}$.

The addition of a second $\mathrm{TiO}_{2}$ layer in the DL model modifies the charge of the original $\mathrm{TiO}_{2} \mathrm{AL}$. We can also calculate the DL structures in order to show that this $\mathrm{TiO}_{2}$ overlayer compensates exactly in all three DL surface structures for this change. For all three surface terminations, we can neglect the bulk net charges as we showed the $\mathrm{TiO}_{2}$ and $\mathrm{SrO}$ layers to compensate for each other before, and therefore, the top three $\mathrm{ALs}$ (i.e., one $\mathrm{SrO}$ and two $\mathrm{TiO}_{2}$ layers) solely remain of interest. Let us start from the $\mathrm{SrO}$ layer and move towards the surface. For all three terminations, the $\mathrm{SrO}$ layer is bulk-coordinated which leads to a net charge of $\sigma a_{0}^{2}=+0.484 e^{-}$per STO unit cell area. The following $\mathrm{TiO}_{2}$ layer is coordinated differently compared to a bulk $\mathrm{TiO}_{2}$ layer, and the coordination depends on the structure of the terminating surface layer. However, the resulting net charge for the $\mathrm{TiO}_{2}$ layer under observation is the same for $(1 \times 1)$, $(2 \times 1)$, and $(2 \times 2)$ domains, and thus $\sigma^{\prime} a_{0}^{2}=-0.242 e^{-}$, already meeting the electrostatic stability criterion. Therefore, the top $\mathrm{TiO}_{2}$ layer should have no net charge in order to be stable. Calculating this net charge for every $\mathrm{TiO}_{2}$ layer of the $(1 \times 1),(2 \times 1)$, and $(2 \times 2)$ individually, we indeed obtain $\sigma^{\prime \prime} a_{0}^{2}=0$ for every termination. As a consequence, the $(1 \times 1),(2 \times 1)$, and $(2 \times 2)$ termination all satisfy the electrostatic requirements. Note, however, that this criterion is met neither by the $\mathrm{Sr}$ adatom model nor the $\mathrm{O}$ overlayer model. If the metal-rich surfaces suggested by our direct methods result in a metallic layer, the required compensation could occur, but the electronic structure of such an overlayer is not known.

\section{CONCLUSIONS}

We have presented a detailed and broad study of the surface structure of the technologically and scientifically important perovskite material $\mathrm{SrTiO}_{3}(001)$ using SXRD, both at room temperature in UHV and at conditions typical for the growth of thin films. The surface was also characterized ex situ by AFM, XPS, and LEIS, and a $\mathrm{TiO}_{2}$ termination was found.

The cold surface simultaneously contains three different terminations, namely, $(2 \times 1)$ and $(2 \times 2)$ reconstructions and a $(1 \times 1)$ relaxation. During the refinement of the cold data set, over 70 models were tested, of which 49 are described in more detail in this work. The atomic coordinates of the final model are given. It consists of a characteristic double $\mathrm{TiO}_{2}$ top layer, whereby the two reconstructions contain a repetition of a distinctive zigzag motif, similar to the one proposed by Erdman et al. for the $(2 \times 1)$ termination. ${ }^{14}$ Both reconstructions are energetically favorable according to our DFT calculations, in agreement with other theoretical work. ${ }^{52,59}$

The hot surface can be modelled using a double-layered 
$\mathrm{TiO}_{2}$ termination, very similar to the cold $(1 \times 1)$, but with more pronounced puckering, and the atomic coordinates are shown. Direct methods analysis using PARADIGM supports the $\mathrm{TiO}_{2}$-rich surface and the atomic positions.

Both the cold and hot surfaces have significant deviations from the high-symmetry starting positions down to a depth of three unit cells. This may have important consequences regarding surface ferroelectricity and other nonlinear properties of the surface.

Surface vibrational energy considerations suggest the possibility of the $(1 \times 1)$ in fact being a temperature-induced disordered mixture of the two types of reconstructions. This would explain the presence of only the $(1 \times 1)$ structure upon heating the sample. Finally, our experimental results are in agreement with electrostatic considerations on the stability of the polar surface of STO.

\section{ACKNOWLEDGMENTS}

Fruitful discussions with R. Feidenhans'l, I. K. Robinson, H. H. Brongersmaa, M. de Ridder, R. ter Veen, K. Peters, J. Krempasky, and L. Patthey are gratefully acknowledged. Support of this work by the Schweizerischer Nationalfonds zur Förderung der wissenschaftlichen Forschung and the staff of the Swiss Light Source is gratefully acknowledged. This work was partly performed at the Swiss Light Source, Paul Scherrer Institut. Work at UWM is supported by the US DOE under contract No. DE-FG02-06ER46277. *philip.willmott@psi.ch

${ }^{1}$ S. Jin, T. H. Tiefel, M. McCormack, R. A. Fastnacht, R. Ramesh, and L. H. Chen, Science 264, 413 (1994).

${ }^{2}$ D. D. Fong, G. B. Stephenson, S. K. Streiffer, J. A. Eastman, O. Auciello, P. H. Fuoss, and C. Thompson, Science 304, 1650 (2004).

${ }^{3}$ C. H. Ahn, K. M. Rabe, and J.-M. Triscone, Science 303, 488 (2004).

${ }^{4}$ A. Ohtomo and H. Y. Hwang, Nature (London) 427, 423 (2004).

${ }^{5}$ J. H. Haeni et al., Nature (London) 430, 758 (2004).

${ }^{6}$ J. E. T. Andersen and P. J. Møller, Appl. Phys. Lett. 56, 1847 (1990).

${ }^{7}$ T. Matsumoto, H. Tanaka, T. Kawai, and S. Kawai, Surf. Sci. 278, L153 (1992).

${ }^{8}$ M. Naito and H. Sato, Physica C 229, 1 (1994).

${ }^{9}$ Q. D. Jiang and J. Zegenhagen, Surf. Sci. 338, L882 (1995).

${ }^{10}$ Q. D. Jiang and J. Zegenhagen, Surf. Sci. 425, 343 (1999).

${ }^{11}$ G. Charlton, S. Brennan, C. A. Muryn, R. McGrath, D. Norman, T. S. Turner, and G. Thornton, Surf. Sci. 457, L376 (2000).

${ }^{12}$ T. Kubo and H. Nozoye, Phys. Rev. Lett. 86, 1801 (2001).

${ }^{13}$ A. Kazimirov, D. M. Goodner, M. J. Bedzyk, J. Bai, and C. R. Hubbard, Surf. Sci. 492, L711 (2001).

${ }^{14}$ N. Erdman, K. R. Poeppelmeier, O. Warschkow, D. E. Ellis, and L. D. Marks, Nature (London) 419, 55 (2002).

${ }^{15}$ M. R. Castell, Surf. Sci. 505, 1 (2002).

${ }^{16}$ M. R. Castell, Surf. Sci. 516, 33 (2002).

${ }^{17}$ K. Johnston, M. R. Castell, A. T. Paxton, and M. W. Finnis, Phys. Rev. B 70, 085415 (2004).

${ }^{18}$ V. Vonk, S. Konings, G. J. van Hummel, S. Harkema, and H. Graafsma, Surf. Sci. 595, 183 (2005).

${ }^{19}$ F. Silly, D. T. Newell, and M. R. Castell, Surf. Sci. 600, L219 (2006)

${ }^{20}$ L. M. Liborio, C. G. Sánchez, A. T. Paxton, and M. W. Finnis, J. Phys.: Condens. Matter 17, L223 (2005).

${ }^{21}$ R. Herger, P. R. Willmott, O. Bunk, C. M. Schlepütz, B. D. Patterson, and B. Delley, Phys. Rev. Lett. 98, 076102 (2007).

${ }^{22} \mathrm{R}$. Herger and K. Peters (private communication).

${ }^{23}$ M. Kawasaki, K. Takahashi, T. Maeda, R. Tsuchiya, M. Shinohara, O. Ishiyama, T. Yonezawa, M. Yoshimoto, and H. Koinuma, Science 266, 1540 (1994).

${ }^{24}$ G. Koster, B. L. Kropman, G. J. H. M. Rijnders, D. H. A. Blank, and H. Rogalla, Appl. Phys. Lett. 73, 2920 (1998).

${ }^{25}$ J. C. Dupin, D. Gonbeau, P. Vinatier, and A. Levasseur, Phys. Chem. Chem. Phys. 2, 1319 (2000).

${ }^{26}$ H. H. Brongersma, M. Draxler, M. de Ridder, and P. Bauer, Surf. Sci. Rep. 62, 63 (2007).

${ }^{27}$ T. Ohnishi, K. Shibuya, M. Lippmaa, D. Kobayashi, H. Kumigashira, M. Oshima, and H. Koinuma, Appl. Phys. Lett. 85, 272 (2004).

${ }^{28}$ B. D. Patterson et al., Nucl. Instrum. Methods Phys. Res. A 540, 42 (2005).

${ }^{29}$ P. R. Willmott et al., Appl. Surf. Sci. 247, 188 (2005).

${ }^{30}$ C. M. Schlepütz, R. Herger, P. R. Willmott, B. D. Patterson, O. Bunk, C. Brönnimann, B. Henrich, G. Hülsen, and E. F. Eikenberry, Acta Crystallogr., Sect. A: Found. Crystallogr. 61, 418 (2005).

${ }^{31}$ K. Szot and W. Speier, Phys. Rev. B 60, 5909 (1999).

${ }^{32}$ E. Vlieg, J. Appl. Crystallogr. 30, 532 (1997).

${ }^{33}$ O. Bunk, Ph.D. thesis, University of Hamburg, Department of Physics, 1999, URL http://www.sub.uni-hamburg.de/opus/ volltexte/1999/99/

${ }^{34}$ W. C. Hamilton, Acta Crystallogr. 18, 502 (1965).

${ }^{35}$ The $R$ factor used here is defined as $R(|F|)=\frac{\left.\sum_{i=1}^{N}|| F\right|_{i, \text { exp }}-|F|_{i, \text { theor }} \mid}{\sum_{i=1}^{N}|F|_{i, \text { exp }}}$, where $|F|$ is the amplitude of the structure factor.

${ }^{36}$ Reduced $\chi_{r}^{2}$, values were used as defined by $\chi_{r}^{2}\left(|F|^{2}\right)$ $=\frac{1}{N-P} \sum_{i=1}^{N} \frac{\left(|F|_{i, \exp }^{2}-|F|_{i, \text { theor }}^{2}\right)^{2}}{\sigma_{|F|_{i, \text { exp }}^{2}}^{2}}$, where $N$ is the number of measured data points, $P$ the number of parameters, $|F|^{2}$ the squared amplitude of the structure factor, i.e., the measured intensity, and $\sigma_{|F|}$, the error of the measured intensity.

${ }^{37} \mathrm{~K}$. Lonsdale, in International Tables for X-Ray Crystallography, edited by C. H. MacGillavry, G. D. Rieck, and K. Lonsdale (Reidel, Dordrecht, 1985), Vol. III, Chap. 3.3.5, p. 232.

${ }^{38}$ K. N. Trueblood, H. B. Burgi, H. Burzlaff, J. D. Dunitz, C. M. Gramaccioli, H. H. Schulz, U. Shmueli, and S. C. Abrahams, Acta Crystallogr., Sect. A: Found. Crystallogr. A52, 770 (1996).

${ }^{39}$ B. Delley, J. Chem. Phys. 92, 508 (1990).

${ }^{40}$ B. Delley, J. Phys. Chem. 100, 6107 (1996).

${ }^{41}$ B. Delley, J. Chem. Phys. 113, 7756 (2000).

${ }^{42}$ J. P. Perdew and Y. Wang, Phys. Rev. B 45, 13244 (1992).

${ }^{43}$ J. P. Perdew, K. Burke, and M. Ernzerhof, Phys. Rev. Lett. 77, 3865 (1996). 
${ }^{44}$ B. Delley, Mol. Simul. 32, 117 (2006).

${ }^{45}$ E. Vlieg, J. Appl. Crystallogr. 33, 401 (2000).

${ }^{46}$ X. Torrelles, J. Rius, F. Boscherini, S. Heun, B. H. Mueller, S. Ferrer, J. Alvarez, and C. Miravitlles, Phys. Rev. B 57, R4281 (1998).

${ }^{47}$ Y. Yacoby, R. Pindak, R. MacHarrie, L. Pfeiffer, L. Berman, and R. Clarke, J. Phys.: Condens. Matter 12, 3929 (2000).

${ }^{48}$ D. K. Saldin, R. J. Harder, V. L. Shneerson, and W. Moritz, J. Phys.: Condens. Matter 13, 10689 (2001).

${ }^{49}$ L. D. Marks, N. Erdman, and A. Subramanian, J. Phys.: Condens. Matter 13, 10677 (2001).

${ }^{50}$ J. R. Fienup, Appl. Opt. 21, 2758 (1982).

${ }^{51}$ C. Noguera, J. Phys.: Condens. Matter 12, R367 (2000).

${ }^{52}$ O. Warschkow, M. Asta, N. Erdman, K. R. Poeppelmeier, D. E. Ellis, and L. D. Marks, Surf. Sci. 573, 446 (2004).

${ }^{53}$ P. N. Keating, Phys. Rev. 145, 637 (1966).

${ }^{54}$ See EPAPS Document No. E-PRBMDO-76-155739 for 3D animations of the final structures and tabulated atomic coordinates in ASCII format. For more information on EPAPS, see http:// www.aip.org/pubservs/epaps.html

${ }^{55}$ Note that the FIT refinement assumed incoherently added domains. For this particular model of the hot data set, however, we also tested coherent domain addition and got almost exactly the same result as for incoherent addition.

${ }^{56}$ D. K. Saldin, R. J. Harder, V. L. Shneerson, and W. Moritz, J. Phys.: Condens. Matter 14, 4087 (2002).

${ }^{57}$ R. Fung, V. L. Shneerson, P. F. Lyman, S. S. Parihar, H. T. Johnson-Steigelman, and D. K. Saldin, J. Phys.: Condens. Matter 63, 239 (2007).

${ }^{58}$ D. K. Saldin and V. L. Shneerson, J. Phys.: Condens. Matter (to be published).

${ }^{59}$ E. Heifets, S. Piskunov, E. A. Kotomin, Y. F. Zhukovskii, and D. E. Ellis, Phys. Rev. B 75, 115417 (2007).
${ }^{60}$ Y. Liang and D. A. Bonnell, Surf. Sci. 310, 128 (1994).

${ }^{61}$ Szot and Speier attribute the presence of droplet-like features in their AFM pictures to the formation of SrO islands (Ref. 31). Note that our AFM image in Fig. 1 shows no such droplet features.

${ }^{62}$ N. Erdman, O. Warschkow, M. Asta, K. R. Poeppelmeier, D. E. Ellis, and L. D. Marks, J. Am. Chem. Soc. 125, 10050 (2003).

${ }^{63}$ M. Gajdardziska-Josifovska, P. A. Crozier, and M. R. McCartney, Surf. Sci. 284, 186 (1993).

${ }^{64}$ K. Fukui and Y. Iwasawa, Surf. Sci. 441, 529 (1999).

${ }^{65}$ L. P. Zhang and U. Diebold, Surf. Sci. 413, 242 (1998).

${ }^{66}$ G. Mariotto, S. Murphy, N. Berdunov, S. F. Ceballos, and I. V. Shvets, Surf. Sci. 564, 79 (2004).

${ }^{67}$ B. D. Patterson, R. Herger, H. H. Brongersma, M. de Ridder, and R. ter Veen (private communication).

${ }^{68}$ Discussions with Calipso B. V. made clear that it is difficult to identify surface impurities and $\mathrm{Sr}$ residuals in the outermost layer by coaxial impact-collision iron scattering spectroscopy, the technique used in the work by Ohnishi and co-workers (Ref. 27 and 67).

${ }^{69}$ L. D. Roelofs, G. Y. Hu, and S. C. Ying, Phys. Rev. B 28, 6369 (1983).

${ }^{70}$ N. Bickel, G. Schmidt, K. Heinz, and K. Müller, Phys. Rev. Lett. 62, 2009 (1989).

${ }^{71}$ V. Ravikumar, D. Wolf, and V. P. Dravid, Phys. Rev. Lett. 74, 960 (1995).

${ }^{72} \mathrm{On}$ a speculative note, the $\mathrm{pm}$, symmetry allowing in-plane movements along the $y$ direction might be a reason for the significantly lower surface energy of the $(2 \times 2)$, compared to the $(2 \times 1)$, reconstruction in our DFT calculations (see Table VI), although the chemical similarity of the reconstructions would not necessarily point to such a distinct energy difference.

${ }^{73}$ P. Tasker, J. Phys. C 12, 4977 (1979).

${ }^{74}$ J. Goniakowski and C. Noguera, Surf. Sci. 365, L657 (1996). 